\title{
Concise Neural Nonaffine Control of Air-Breathing Hypersonic Vehicles Subject to Parametric Uncertainties
}

\author{
Xiangwei Bu, ${ }^{1}$ Qiong Wang, ${ }^{2}$ Yan Zhao, ${ }^{1}$ and Guangjun $\mathrm{He}^{1}$ \\ ${ }^{1}$ Air and Missile Defense College, Air Force Engineering University, Xi'an 710051, China \\ ${ }^{2}$ Science College, Air Force Engineering University, Xi'an 710051, China \\ Correspondence should be addressed to Xiangwei Bu; buxiangwei1987@126.com
}

Received 26 March 2017; Revised 11 July 2017; Accepted 14 August 2017; Published 1 October 2017

Academic Editor: Paul Williams

Copyright (c) 2017 Xiangwei $\mathrm{Bu}$ et al. This is an open access article distributed under the Creative Commons Attribution License, which permits unrestricted use, distribution, and reproduction in any medium, provided the original work is properly cited.

\begin{abstract}
In this paper, a novel simplified neural control strategy is proposed for the longitudinal dynamics of an air-breathing hypersonic vehicle (AHV) directly using nonaffine models instead of affine ones. For the velocity dynamics, an adaptive neural controller is devised based on a minimal-learning parameter (MLP) technique for the sake of decreasing computational loads. The altitude dynamics is rewritten as a pure feedback nonaffine formulation, for which a novel concise neural control approach is achieved without backstepping. The special contributions are that the control architecture is concise and the computational cost is low. Moreover, the exploited controller possesses good practicability since there is no need for affine models. The semiglobally uniformly ultimate boundedness of all the closed-loop system signals is guaranteed via Lyapunov stability theory. Finally, simulation results are presented to validate the effectiveness of the investigated control methodology in the presence of parametric uncertainties.
\end{abstract}

\section{Introduction}

Air-breathing hypersonic vehicles (AHVs) are regarded as a promising technology for cost-efficient and reliable access to space. One of the major issues with respect to the developments of AHVs is to maintain controllability of the vehicle during various phases of flight [1-3]. However, flight control design for such vehicles is very challenging due to significant uncertainties, strong couplings, nonminimum phase characteristics, and significant flexible effects [4-7].

Recently, many classic control methodologies have been proposed for the longitudinal dynamics of AHVs. To guarantee the robustness with respect to parametric uncertainties, an adaptive sliding mode controller is addressed for an AHV $[8,9]$. The starting point of that strategy $[8,9]$ is to linearize the vehicle model as an affine one, which is required for the control design. In [10], a continuous highorder sliding mode control scheme is investigated using an affine AHV model. In that study, the undesired chattering phenomenon is avoided owing to the continuous control inputs. For an AHV with actuator faults, an adaptive faulttolerant controller is devised on the basis of a TakagiSugeno (T-S) fuzzy system [11]. In [12, 13], a linear parameter-varying (LPV) affine model is first constructed to describe the complex longitudinal dynamics of an AHV. Then, a nonfragile switching tracking control methodology is proposed $[12,13]$. In [14], a compound control approachcombined trajectory linearization control (TLC) with active disturbance rejection control (ADRC) is presented for an AHV. Moreover, the robustness of that strategy is enhanced via linear-extended state observers [14]. For the longitudinal dynamics of an AHV, a new tracking control scheme is exploited by solving a system of linear algebraic equations [15]. In [16], a robust controller is explored for an $\mathrm{AHV}$, and moreover, a new nonlinear disturbance observer (NDO) is developed for robustness enhancement.

It is noted that the altitude dynamics of AHVs can be rewritten as a strict feedback affine model based on strict 
assumptions, which makes backstepping control achievable [17-20]. In [1], a robust adaptive control method is studied via backstepping. However, the smooth parameter projection utilized in that paper may result in high computational loads because of too many adaptive parameters. Meanwhile, the problem of "explosion of terms" is ignored [1]. In [19], a new tracking differentiator is exploited to provide estimations of the time derivatives of virtual controllers while the problem of "explosion of terms" is eliminated. Based on the results of [21], a new NDO is constructed, which guarantees that the proposed backstepping controller can provide robust tracking of velocity and altitude reference trajectories in the presence of parametric uncertainties and external disturbances [19]. It has been demonstrated that neural networks (NNs) have the ability of global approximation [22-25]. Thus, improved backstepping control methodologies with robust performance can be achieved if the unknown nonlinearities are approximated by NNs [26-29]. To reduce the computational cost of neural approximation, the NNs are employed to approach the devised backstepping controllers rather than unknown functions while a nonsingular direct neural control strategy is addressed for an AHV [30]. Furthermore, a novel control scheme with low computational loads is exploited for an AHV based on a pure feedback affine model [31]. Meanwhile, the learning parameters are decreased via a minimal-learning parameter (MLP) method [31].

It is worth pointing out that all the above results are obtained based on affine models. That is, it requires that the AHV model has to be linear in the control inputs. However, in fact, the AHV model has no linear appearance of the control inputs $[32,33]$. On the basis of the previous approaches, we have to first transform the AHV model into an affine formulation and then exploit controllers utilizing linearized or affine AHV models. In this paper, we propose a novel adaptive neural controller for the longitudinal dynamics of an AHV without using affine models. The vehicle dynamics is decomposed as the velocity subsystem and the altitude subsystem to be controlled separately. For both subsystems, simplified neural controllers with low computational loads are addressed via an MLP technique. By using a novel model transformation method, the altitude dynamics is rewritten as a pure feedback formulation. Hence, a simplified design is achieved. Finally, the effectiveness of the studied methodology is verified by simulation results. The special contributions of this paper are summarized as follows:

(1) Different from the existing studies, the exploited controller is directly derived from nonaffine models. Moreover, the strict requirements [30] that the unknown functions have to be strictly positive and bounded are released in this paper. Thereby, the proposed control scheme possesses excellent practicability and reliability.

(2) Compared with backstepping control strategies $[18-20,30]$, the explored controller exhibits a more concise structure and a lower computational load.

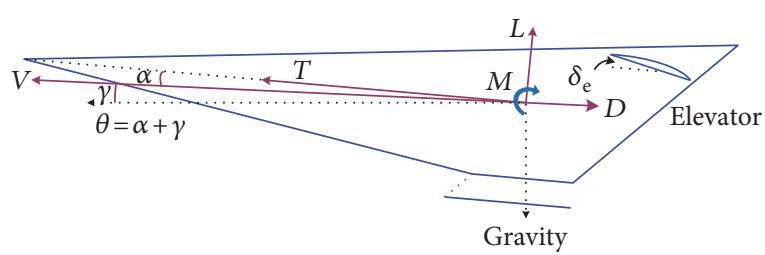

Figure 1: The geometry and force map of an AHV.

In this study, there is no need of a complicated recursive design process of backstepping. In comparison with the neural backstepping control approach [30], much less controllers, NNs, and learning parameters are needed in this study.

The remainder of this paper is structured as follows. The vehicle model and preliminaries are shown in Section 2. Section 3 presents the control design process. The simulation studies are made in Section 4, and the conclusions are proposed in Section 5.

\section{AHV Model and Preliminaries}

2.1. The AHV Model and Control Goal. In this paper, we consider a longitudinal dynamic model of an AHV developed by Bolender and Doman [32]. The AHV's longitudinal sketch and force map are shown in Figure 1. The equations of motion are constructed by applying Lagrange's equations. Moreover, the vehicle is viewed as a single flexible structure with mass-normalized mode shapes. Thus, the vehicle model contains flexible effects. The equations of motion are expressed as follows [33]:

$$
\begin{aligned}
\dot{V} & =\frac{T \cos (\theta-\gamma)-D}{m}-g \sin \gamma, \\
\dot{h} & =V \sin \gamma, \\
\dot{\gamma} & =\frac{L+T \sin (\theta-\gamma)}{m V}-\frac{g \cos \gamma}{V}, \\
\dot{\theta} & =Q, \\
\dot{Q} & =\frac{M+\widetilde{\psi}_{1} \ddot{\eta}_{1}+\widetilde{\psi}_{2} \ddot{\eta}_{2}}{I_{y y}}, \\
k_{1} \ddot{\eta}_{1} & =-2 \zeta_{1} \omega_{1} \dot{\eta}_{1}-\omega_{1}^{2} \eta_{1}+N_{1}-\widetilde{\psi}_{1} \frac{M}{I_{y y}}-\frac{\widetilde{\psi}_{1} \widetilde{\psi}_{2} \ddot{\eta}_{2}}{I_{y y}}, \\
k_{2} \ddot{\eta}_{2} & =-2 \zeta_{2} \omega_{2} \dot{\eta}_{2}-\omega_{2}^{2} \eta_{2}+N_{2}-\widetilde{\psi}_{2} \frac{M}{I_{y y}}-\frac{\widetilde{\psi}_{2} \widetilde{\psi}_{1} \ddot{\eta}_{1}}{I_{y y}},
\end{aligned}
$$


where the approximations of $T, D, L, M, N_{1}$, and $N_{2}$ are designed as follows [33]:

$$
\begin{aligned}
T & \approx C_{T}^{\alpha^{3}} \alpha^{3}+C_{T}^{\alpha^{2}} \alpha^{2}+C_{T}^{\alpha} \alpha+C_{T}^{0}, \\
D & \approx \bar{q} S\left(C_{D}^{\alpha^{2}} \alpha^{2}+C_{D}^{\alpha} \alpha+C_{D}^{\delta_{\mathrm{e}}^{2}} \delta_{\mathrm{e}}^{2}+C_{D}^{\delta_{\mathrm{e}}} \delta_{\mathrm{e}}+C_{D}^{0}\right), \\
L & \approx \bar{q} S\left(C_{L}^{\alpha} \alpha+C_{L}^{\delta_{\mathrm{e}}} \delta_{\mathrm{e}}+C_{L}^{0}\right), \\
M & \approx z_{T} T+\bar{q} S \bar{c}\left[C_{M, \alpha}^{\alpha^{2}} \alpha^{2}+C_{M, \alpha}^{\alpha} \alpha+C_{M, \alpha}^{0}+c_{\mathrm{e}} \delta_{\mathrm{e}}\right], \\
N_{1} & \approx N_{1}^{\alpha^{2}} \alpha^{2}+N_{1}^{\alpha} \alpha+N_{1}^{0}, \\
N_{2} & \approx N_{2}^{\alpha^{2}} \alpha^{2}+N_{2}^{\alpha} \alpha+N_{2}^{\delta_{\mathrm{e}}} \delta_{\mathrm{e}}+N_{2}^{0}, \\
\bar{q} & =\frac{1}{2} \rho V^{2}, \quad \rho=\rho_{0} \exp \left(-\frac{h-h_{0}}{h_{s}}\right),
\end{aligned}
$$

with

$$
\begin{aligned}
& C_{T}^{\alpha^{3}}=\beta_{1}(h, \bar{q}) \Phi+\beta_{2}(h, \bar{q}), \\
& C_{T}^{\alpha^{2}}=\beta_{3}(h, \bar{q}) \Phi+\beta_{4}(h, \bar{q}), \\
& C_{T}^{\alpha}=\beta_{5}(h, \bar{q}) \Phi+\beta_{6}(h, \bar{q}), \\
& C_{T}^{0}=\beta_{7}(h, \bar{q}) \Phi+\beta_{8}(h, \bar{q}) .
\end{aligned}
$$

The above model is composed of five rigid body states (i.e., $V, h, \gamma, \theta$, and $Q$ ), four flexible states (i.e., $\eta_{1}, \dot{\eta}_{1}, \eta_{2}$, and $\dot{\eta}_{2}$ ), and two control inputs (i.e., $\Phi$ and $\delta_{\mathrm{e}}$ ). It is noticed that the control inputs do not occur explicitly in (1), (2), (3), (4), (5), (6), and (7). However, they appear through $T, D, L$, $M, N_{1}$, and $N_{2}$. For more detailed definitions of the model parameters and coefficients, the reader could refer to [33] or the Nomenclature.

The control goal pursued in this study is to devise adaptive neural controllers for an AHV without using affine modes such that velocity $V$ and altitude $h$ track their reference trajectories $V_{\text {ref }}$ and $h_{\text {ref }}$, respectively. Meanwhile, all the closed-loop system signals are bounded and the proposed controller is robust to parametric uncertainties.

2.2. NN Description. To guarantee the controller's robustness, we employ the radial basis function NN (RBFNN) to approximate the lumped uncertainties of both subsystems of AHVs. The RBFNN is defined as the mapping relationship between the input vector $\mathbf{X}=\left[\ell_{1}, \ell_{2}, \ldots, \ell_{n}\right]^{T} \in \mathbf{R}^{n}$ and the output $y \in \mathbf{R}$ [34].

$$
y=\mathbf{W}^{T} \mathbf{h}(\mathbf{X}),
$$

where $\mathbf{W}=\left[w_{1}, w_{2}, \ldots, w_{p}\right]^{T} \in \mathbf{R}^{p}$ denotes a weight vector; $p$ and $n$ represent the node number and input number, respectively; $\mathbf{h}(\mathbf{X})=\left[h_{1}(\mathbf{X}), h_{2}(\mathbf{X}), \ldots, h_{p}(\mathbf{X})\right]^{T} \in \mathbf{R}^{p}$ with $h_{j}(\mathbf{X})$ is defined as follows:

$$
h_{j}(\mathbf{X})=\exp \left(-\frac{\|\mathbf{X}-\mathbf{c}\|^{2}}{2 b_{j}^{2}}\right), \quad j=1,2, \ldots, p,
$$

where $\mathbf{c}=\left[c_{1}, c_{2}, \ldots, c_{n}\right]^{T} \in \mathbf{R}^{n}$ and $\mathbf{b}=\left[b_{j 1}, b_{j 2}, \ldots, b_{j n}\right]^{T} \in \mathbf{R}^{n}$ mean a center vector and a width vector of $h_{j}(\mathbf{X})$, respectively.
For an arbitrary continuous unknown function $F(\mathbf{X})$, it has be proved that there exists an ideal weight vector $\mathbf{W}^{*}=\left[w_{1}^{*}, w_{2}^{*}, \ldots, w_{p}^{*}\right]^{T} \in \mathbf{R}^{p}$ such that $[34]$

$$
F(\mathbf{X})=\mathbf{W}^{* T} \mathbf{h}(\mathbf{X})+\varepsilon, \quad|\varepsilon| \leq \varepsilon_{M},
$$

where $\varepsilon$ and $\varepsilon_{M}$ are the approximation error and its upper bound, respectively. Noting that $\mathbf{W}^{*}$ is completely unknown, its elements $w_{1}^{*}, w_{2}^{*}, \ldots, w_{p}^{*}$ are required to be adjusted adaptively. In what follows, we define $\varphi=\left\|\mathbf{W}^{*}\right\|^{2}$ and regulate $\varphi$ rather than $w_{1}^{*}, w_{2}^{*}, \ldots, w_{p}^{*}$ to achieve satisfactory neural approximation. In this way, the learning parameters are decreased greatly such that the computational burden is quite low. This is the so-called MLP scheme.

2.3. High-Order Tracking Differentiator. In the subsequent developments, we will employ a high-order tracking differentiator (HTD) [35] to estimate the newly defined states. This HTD is formulated as follows:

$$
\begin{aligned}
& \dot{\chi}_{1}=\chi_{2}, \\
& \dot{\chi}_{2}=\chi_{3}, \\
& \dot{\chi}_{3}=\chi_{4}, \\
& \dot{\chi}_{4}=-R^{4}\left[a_{1}\left(\chi_{1}-v\right)+a_{2} \frac{\chi_{2}}{R}+a_{3} \frac{\chi_{3}}{R^{2}}+a_{4} \frac{\chi_{4}}{R^{3}}\right],
\end{aligned}
$$

where $R, a_{1}, a_{2}, a_{3}$, and $a_{4}$ are positive constants to be chosen; $v$ is the input signal; $\chi_{1}, \chi_{2}, \chi_{3}$, and $\chi_{4}$ denote the states of the HTD and they stand for the estimations of $v, \dot{v}$, $\ddot{v}$, and $\dddot{v}$, respectively. The relative estimation errors are defined as follows: $e_{1}=\chi_{1}-v, e_{2}=\chi_{2}-\dot{v}, e_{3}=\chi_{3}-\ddot{v}$, and $e_{4}=\chi_{4}-\dddot{v}$. By choosing an infinitely large $R$ for the HTD, we have

$$
\begin{aligned}
& \lim _{R \rightarrow+\infty}\left(\chi_{1}-v\right)=\lim _{R \rightarrow+\infty} e_{1}=0, \\
& \frac{d \lim _{R \rightarrow+\infty}\left(\chi_{1}-v\right)}{d t}=\lim _{R \rightarrow+\infty}\left(\dot{\chi}_{1}-\dot{v}\right)=\lim _{R \rightarrow+\infty}\left(\chi_{2}-\dot{v}\right)=\lim _{R \rightarrow+\infty} e_{2}=0, \\
& \frac{d \lim _{R \rightarrow+\infty}\left(\chi_{2}-\dot{v}\right)}{d t}=\lim _{R \rightarrow+\infty}\left(\dot{\chi}_{2}-\ddot{v}\right)=\lim _{R \rightarrow+\infty}\left(\chi_{3}-\ddot{v}\right)=\lim _{R \rightarrow+\infty} e_{3}=0, \\
& \frac{d \lim _{R \rightarrow+\infty}\left(\chi_{3}-\ddot{v}\right)}{d t}=\lim _{R \rightarrow+\infty}\left(\dot{\chi}_{3}-\dddot{v}\right)=\lim _{R \rightarrow+\infty}\left(\chi_{4}-\dddot{v}\right)=\lim _{R \rightarrow+\infty} e_{4}=0 .
\end{aligned}
$$

Remark 1. It is obvious that the estimation errors $e_{i}(i=1$, $2,3,4)$ can converge to zero by designing an adequately large $R$. In choosing an infinitely large but bounded $R$ for the THD in simulation study, we know that there exist positive constants $\bar{e}_{i}(i=1,2,3,4)$ such that $\bar{e}_{i} \geq\left|e_{i}\right|(i=1,2,3,4)[23]$.

2.4. Preliminaries. To start the control design, the following preliminaries are required.

Definition 1 (see [36]). For any compact set $\Omega_{0}$, if there exist a positive parameter $\Sigma$ and $I=I\left(\Sigma, Y_{0}\left(t_{0}\right)\right)$ such that $\left\|Y_{0}(t)\right\| \leq \Sigma$ for all $Y\left(t_{0}\right) \in \Omega_{0}$ and $t \geq t_{0}+I$, then $Y_{0}(t)$ is semiglobally uniformly ultimately bounded. 
Lemma 1 (implicit function theorem, see [36, 37]). The implicit function $H: \mathbf{R}^{l} \times \mathbf{R}^{r} \rightarrow \mathbf{R}^{l}$ is continuously differentiable at each $(\boldsymbol{\omega}, \boldsymbol{\sigma})$ of an open set $Y \subset \mathbf{R}^{l} \times \mathbf{R}^{r}$. Define $\left(\boldsymbol{\Phi}_{0}, \boldsymbol{\sigma}_{0}\right)$ as a point in $Y$ for which $H\left(\boldsymbol{\Phi}_{0}, \sigma_{0}\right)=0$ and the Jacobian matrix $(\partial H / \partial \varpi)\left(\boldsymbol{\Phi}_{0}, \boldsymbol{\sigma}_{0}\right)$ is nonsingular. Then, there are neighborhoods $U \subset \mathbf{R}^{l}$ of $\boldsymbol{\Phi}_{0}$ and $G \subset \mathbf{R}^{r}$ of $\boldsymbol{\sigma}_{0}$ such that for each $\boldsymbol{\sigma} \in G$, the equation $H(\boldsymbol{\omega}, \boldsymbol{\sigma})=0$ has a unique solution $\varpi \in U$. Furthermore, the solution can be expressed as $\boldsymbol{\omega}=g_{0}$ $(\boldsymbol{\sigma})$, where $g_{0}(\cdot)$ is a continuously differentiable function at $\sigma=\sigma_{0}$.

Remark 2. For the detailed proof of Lemma 1, the reader could refer to [36]. It is noted that if the implicit function $H(\boldsymbol{\Phi}, \boldsymbol{\sigma})$ satisfies Lemma $1, H(\boldsymbol{\Phi}, \boldsymbol{\sigma})$ can be viewed as a function of $\sigma$ since there exists a function $g_{0}(\cdot)$ such that $\boldsymbol{\omega}=g_{0}(\boldsymbol{\sigma})$.

\section{Controller Design}

According to the discussions presented in $[1,7,19,20]$, we know that the vehicle dynamics can be reasonably decomposed as the velocity subsystem (i.e., (1)) and the altitude subsystem (i.e., (2), (3), (4), and (5)).

3.1. Velocity Controller Design. In this subsection, the objective is to devise a neural controller $\Phi$ for the velocity subsystem by applying a nonaffine model such that velocity $V$ follows its reference command $V_{\text {ref }}$.

The velocity subsystem can be expressed as the following nonaffine model:

$$
\dot{V}=f_{V}(V, \Phi),
$$

where $f_{V}(V, \Phi)$ is a completely unknown and continuously differentiable function.

Assumption 1. The following inequality

$$
\frac{\partial f_{V}(V, \Phi)}{\partial \Phi}>0
$$

holds for all $(V, \Phi) \in \Omega_{V} \times \mathbf{R}$ with a controllability region $\Omega_{V}$.

Remark 3. According to [33] and the response bounds of the rigid-body states, we conclude that Assumption 1 is satisfied.

Define velocity tracking error as follows:

$$
\tilde{V}=V-V_{\text {ref }} .
$$

Taking time derivative along (17) and substituting (15) lead to

$$
\dot{\tilde{V}}=\dot{V}-\dot{V}_{\text {ref }}=k_{V} \Phi+F_{V}(V, \Phi)-\dot{V}_{\text {ref }},
$$

where $k_{V}>0$ is a design parameter; $F_{V}(V, \Phi)=f_{V}(V, \Phi)$ $k_{V} \Phi$ denotes the lumped uncertainty of the velocity subsystem.
The control effort $\Phi$ is chosen as follows:

$$
\Phi=k_{V}^{-1}\left(\Phi_{0}-\Phi_{1}\right)
$$

where $\Phi_{0}=-k_{V 1} \tilde{V}-k_{V 2} \int_{0}^{t} \tilde{V} d \tau+\dot{V}_{\text {ref }}$ with $k_{V 1}>0$ and $k_{V 2}>0 ; \Phi_{1}$ is a neural control signal designed to cancel $F_{V}(V, \Phi)$ by employing an RBFNN.

Invoking Lemma 1, we have that there exists $\Phi_{1}^{*}$ such that

$$
H_{1}\left(V, \Phi_{0}, \Phi_{1}^{*}\right) \triangleq F_{V}\left(V, k_{V}^{-1}\left(\Phi_{0}-\Phi_{1}^{*}\right)\right)-\Phi_{1}^{*}=0 .
$$

Then, we obtain the following theorem.

Theorem 1. Define

$$
k_{V}>\frac{1}{2} \frac{\partial f_{V}(V, \Phi)}{\partial \Phi} .
$$

Then there exist a set $\Omega_{V} \subset \mathbf{R}$ and a unique $\Phi_{1}^{*}$ that is a function of $V$ and $\Phi_{0}$ such that $\Phi_{1}^{*}\left(V, \Phi_{0}\right)$ satisfies (20) for all $\left(V, \Phi_{0}\right) \in \Omega_{V} \times \mathbf{R}$.

Proof. The sufficient condition for the existence of $\Phi_{1}^{*}$ is that the following inequality holds $[37,38]$ :

$$
\left|\frac{\partial F_{V}(V, \Phi)}{\partial \Phi_{1}^{*}}\right|<1
$$

Considering (16), (19), and (21) and the fact that $F_{V}$ $(V, \Phi)=f_{V}(V, \Phi)-k_{V} \Phi$, we obtain

$$
\begin{aligned}
\left|\frac{\partial F_{V}(V, \Phi)}{\partial \Phi_{1}^{*}}\right| & =\left|\frac{\partial}{\partial \Phi_{1}^{*}}\left[f_{V}(V, \Phi)-k_{V} \Phi\right]\right| \\
& =\left|\frac{\partial\left[f_{V}(V, \Phi)-k_{V} \Phi\right]}{\partial \Phi} \frac{\partial \Phi}{\partial \Phi_{1}^{*}}\right| \\
& =\left|\left[\frac{\partial f_{V}(V, \Phi)}{\partial \Phi}-k_{V}\right] \frac{1}{k_{V}}\right| \\
& =\left|\frac{1}{k_{V}} \frac{\partial f_{V}(V, \Phi)}{\partial \Phi}-1\right|<1 .
\end{aligned}
$$

It is clear that $\Phi_{1}^{*}$ exists.

Furthermore, notice that

$$
\begin{aligned}
\frac{\partial}{\partial \Phi_{1}^{*}} H_{1}\left(V, \Phi_{0}, \Phi_{1}^{*}\right) & =\frac{\partial}{\partial \Phi_{1}^{*}}\left[F_{V}\left(V, \Phi^{*}\right)-\Phi_{1}^{*}\right] \\
& =\frac{\partial}{\partial \Phi^{*}}\left[f_{V}\left(V, \Phi^{*}\right)-k_{V} \Phi^{*}\right] \frac{\partial \Phi^{*}}{\partial \Phi_{1}^{*}}-1 \\
& =\left[\frac{\partial}{\partial \Phi^{*}} f_{V}\left(V, \Phi^{*}\right)-k_{V}\right]\left(-\frac{1}{k_{V}}\right)-1 \\
& =-\frac{1}{k_{V}} \frac{\partial f_{V}\left(V, \Phi^{*}\right)}{\partial \Phi^{*}},
\end{aligned}
$$

with $\Phi^{*}=k_{V}^{-1}\left(\Phi_{0}-\Phi_{1}^{*}\right)$. 
By (16) and (21), we know that $\partial H_{1}\left(V, \Phi_{0}, \Phi_{1}^{*}\right) /\left(\partial \Phi_{1}^{*}\right)$ is nonsingular. Thus, Theorem 1 holds. This is the end of proof.

Theorem 1 reveals that $\Phi$ is a function of $V$ and $\Phi_{0}$ for all $\left(V, \Phi_{0}\right) \in \Omega_{V} \times \mathbf{R}$ due to $\Phi=k_{V}^{-1}\left(\Phi_{0}-\Phi_{1}\right)$. Thereby, $F_{V}(V, \Phi)=f_{V}(V, \Phi)-k_{V} \Phi$ also is a function of $V$ and $\Phi_{0}$. Define $\mathbf{X}_{1}=\left[V, \Phi_{0}\right]^{T} \in \mathbf{R}^{2}$ as an input vector. Then we introduce an RBFNN to approximate $F_{V}(V, \Phi)$.

$$
F_{V}(V, \Phi)=\mathbf{W}_{1}^{* T} \mathbf{h}_{1}\left(\mathbf{X}_{1}\right)+\iota_{1},\left|\iota_{1}\right| \leq \iota_{1 M},
$$

where $\mathbf{W}_{1}^{*}=\left[w_{11}^{*}, w_{12}^{*}, \ldots, w_{1 p_{1}}^{*}\right]^{T} \in \mathbf{R}^{p_{1}}$ is an ideal weight vector; $\iota_{1}$ and $\iota_{1 M}$ are the approximation error and its upper bound, respectively; $\mathbf{h}_{1}\left(\mathbf{X}_{1}\right)=\left[h_{11}\left(\mathbf{X}_{1}\right), h_{12}\left(\mathbf{X}_{1}\right), \ldots\right.$, $\left.h_{1 p_{1}}\left(\mathbf{X}_{1}\right)\right]^{T} \in \mathbf{R}^{p_{1}}, h_{1 j}\left(\mathbf{X}_{1}\right)\left(j=1,2, \ldots, p_{1}\right)$ have similar formulations to (11).

Define $\varphi_{1}=\left\|\mathbf{W}_{1}^{*}\right\|^{2}$ and choose

$$
\Phi_{1}=\frac{1}{2} \tilde{V} \widehat{\varphi}_{1} \mathbf{h}_{1}^{T}\left(\mathbf{X}_{1}\right) \mathbf{h}_{1}\left(\mathbf{X}_{1}\right)
$$

where $\widehat{\varphi}_{1}$ is the estimation of $\varphi_{1}$ with the following adaptive law:

$$
\dot{\hat{\varphi}}_{1}=\frac{\lambda_{1}}{2} \tilde{V}^{2} \mathbf{h}_{1}^{T}\left(\mathbf{X}_{1}\right) \mathbf{h}_{1}\left(\mathbf{X}_{1}\right)-2 \widehat{\varphi}_{1}
$$

where $\lambda_{1}>0$ is a design parameter.

Theorem 2. Consider the closed-loop system consisting of plant (15) under Assumption 1 with controller (19) and adaptive law (27). Then all the signals involved are semiglobally uniformly ultimately bounded.

Proof. Define

$$
\widetilde{\varphi}_{1}=\widehat{\varphi}_{1}-\varphi_{1}
$$

Substituting (19), (25), and (26) into (18) yields

$$
\begin{aligned}
\dot{\tilde{V}}= & \Phi_{0}-\Phi_{1}+\mathbf{W}_{1}^{* T} \mathbf{h}_{1}\left(\mathbf{X}_{1}\right)+\iota_{1}-\dot{V}_{\text {ref }} \\
= & -k_{V 1} \tilde{V}-k_{V 2} \int_{0}^{t} \tilde{V} d \tau-\frac{1}{2} \tilde{V} \hat{\varphi}_{1} \mathbf{h}_{1}^{T}\left(\mathbf{X}_{1}\right) \mathbf{h}_{1}\left(\mathbf{X}_{1}\right) \\
& +\mathbf{W}_{1}^{* T} \mathbf{h}_{1}\left(\mathbf{X}_{1}\right)+\iota_{1} .
\end{aligned}
$$

The Lyapunov function candidate is chosen as follows:

$$
W_{V}=\frac{\tilde{V}^{2}}{2}+\frac{k_{V 2}}{2}\left(\int_{0}^{t} \tilde{V} d \tau\right)^{2}+\frac{\tilde{\varphi}_{1}^{2}}{2 \lambda_{1}} .
$$

Invoking (27), (28), and (29), the time derivative of $W_{V}$ is derived as follows:

$$
\begin{aligned}
\dot{\mathbf{W}}_{V}= & \tilde{V} \dot{\tilde{V}}+k_{V 2} \tilde{V} \int_{0}^{t} \tilde{V} d \tau+\frac{\widetilde{\varphi}_{1} \dot{\hat{\varphi}}_{1}}{\lambda_{1}} \\
= & -k_{V 1} \tilde{V}^{2}-\frac{1}{2} \tilde{V}^{2} \varphi_{1} \mathbf{h}_{1}^{T}\left(\mathbf{X}_{1}\right) \mathbf{h}_{1}\left(\mathbf{X}_{1}\right)+\tilde{V} \mathbf{W}_{1}^{* T} \mathbf{h}_{1}\left(\mathbf{X}_{1}\right) \\
& +\tilde{V} \iota_{1}-\frac{2 \widetilde{\varphi}_{1} \widehat{\varphi}_{1}}{\lambda_{1}}
\end{aligned}
$$

Due to $\widetilde{\varphi}_{1}^{2}+2 \widetilde{\varphi}_{1}\left(\widehat{\varphi}_{1}-\widetilde{\varphi}_{1}\right)+\varphi_{1}^{2}=\widetilde{\varphi}_{1}^{2}+2 \widetilde{\varphi}_{1} \varphi_{1}+\varphi_{1}^{2}=$ $\left(\widetilde{\varphi}_{1}+\varphi_{1}\right)^{2} \geq 0$, it follows that $2 \widetilde{\varphi}_{1} \widehat{\varphi}_{1} \geq \widetilde{\varphi}_{1}^{2}-\varphi_{1}^{2}$. Thus, the following inequality holds:

$$
\begin{aligned}
\dot{W}_{V} \leq & -k_{V 1} \tilde{V}^{2}-\frac{\widetilde{\varphi}_{1}^{2}}{\lambda_{1}}-\frac{1}{2} \tilde{V}^{2} \varphi_{1} \mathbf{h}_{1}^{T}\left(\mathbf{X}_{1}\right) \mathbf{h}_{1}\left(\mathbf{X}_{1}\right) \\
& +\tilde{V} \mathbf{W}_{1}^{* T} \mathbf{h}_{1}\left(\mathbf{X}_{1}\right)+\tilde{V} \iota_{1}+\frac{\varphi_{1}^{2}}{\lambda_{1}}
\end{aligned}
$$

Notice that

$$
\begin{aligned}
\tilde{V} \mathbf{W}_{1}^{* T} \mathbf{h}_{1}\left(\mathbf{X}_{1}\right) & \leq \frac{\tilde{V}^{2}}{2}\left\|\mathbf{W}_{1}^{* T} \mathbf{h}_{1}\left(\mathbf{X}_{1}\right)\right\|^{2}+\frac{1}{2} \\
& =\frac{\tilde{V}^{2}}{2}\left\|\mathbf{W}_{1}^{*}\right\|^{2}\left\|\mathbf{h}_{1}\left(\mathbf{X}_{1}\right)\right\|^{2}+\frac{1}{2} \\
& =\frac{\tilde{V}^{2}}{2} \varphi_{1} \mathbf{h}_{1}^{T}\left(\mathbf{X}_{1}\right) \mathbf{h}_{1}\left(\mathbf{X}_{1}\right)+\frac{1}{2}, \\
\tilde{V}_{\iota_{1}} & \leq \frac{1}{2} \tilde{V}^{2} \iota_{1 M}^{2}+\frac{1}{2} .
\end{aligned}
$$

Inequality (32) becomes

$$
\dot{W}_{V} \leq-\left(k_{V 1}-\frac{1}{2} l_{1 M}^{2}\right) \tilde{V}^{2}-\frac{\widetilde{\varphi}_{1}^{2}}{\lambda_{1}}+1+\frac{\varphi_{1}^{2}}{\lambda_{1}} .
$$

Let $k_{V 1}>t_{1 M}^{2} / 2$ and define the following compact sets:

$$
\begin{gathered}
\Omega_{\tilde{V}}=\left\{\tilde{V}|| \tilde{V} \mid \leq \sqrt{\left.\frac{1+\left(\varphi_{1}^{2} / \lambda_{1}\right)}{k_{V 1}-(1 / 2) \iota_{1 M}^{2}}\right\},}\right. \\
\Omega_{\tilde{\varphi}_{1}}=\left\{\widetilde{\varphi}_{1}|| \widetilde{\varphi}_{1} \mid \leq \sqrt{\frac{1+\left(\varphi_{1}^{2} / \lambda_{1}\right)}{\left(1 / \lambda_{1}\right)}}\right\} .
\end{gathered}
$$

It can be seen that $\dot{W}_{V}$ will be negative if $\tilde{V} \notin \Omega_{\tilde{V}}$ or $\widetilde{\varphi}_{1} \notin \Omega_{\tilde{\varphi}_{1}}$. Hence, $\tilde{V}$ and $\widetilde{\varphi}_{1}$ are semiglobally uniformly ultimately bounded. This completes the proof. 
3.2. Altitude Controller Design. In this subsection, we will explore a simplified neural controller $\delta_{\mathrm{e}}$ for the altitude subsystem without using backstepping such that altitude $h$ tracks its command $h_{\text {ref }}$.

Define altitude tracking error as $\tilde{h}=h-h_{\text {ref }}$. The command of $\gamma$ is chosen as follows:

$$
\gamma_{d}=\arcsin \left(\frac{-k_{\gamma} \tilde{h}+\dot{h}_{\mathrm{ref}}}{V}\right) \text {, }
$$

where $k_{\gamma}>0$ is a design parameter. If $\gamma$ converges to $\gamma_{d}$, then we have that $h$ converges to $h_{\text {ref }}[26]$. Hence, the subsequent control goal is to let $\gamma$ converge to $\gamma_{d}$.

Define $x_{1}=\gamma, x_{2}=\theta, x_{3}=Q$. Then, a nonaffine model can be derived from the rest of the altitude subsystem (i.e., (3), (4), and (5)).

$$
\begin{aligned}
& \dot{x}_{1}=f_{1}\left(x_{1}, x_{2}\right), \\
& \dot{x}_{2}=x_{3}, \\
& \dot{x}_{3}=f_{3}\left(\mathbf{x}, \delta_{\mathrm{e}}\right),
\end{aligned}
$$

with $\mathbf{x}=\left[x_{1}, x_{2}, x_{3}\right]^{T}$. In (37), $f_{1}\left(x_{1}, x_{2}\right)$ and $f_{3}\left(\mathbf{x}, \delta_{\mathrm{e}}\right)$ are completely unknown and continuously differentiable functions.

Assumption 2. The following are inequalities:

$$
\begin{aligned}
& \frac{\partial f_{1}\left(x_{1}, x_{2}\right)}{\partial x_{2}}>0, \\
& \frac{\partial f_{3}\left(\mathbf{x}, \delta_{\mathrm{e}}\right)}{\partial \delta_{\mathrm{e}}}>0 .
\end{aligned}
$$

The inequalities above are satisfied for all $\left(\mathbf{x}, \delta_{\mathrm{e}}\right) \in \Omega_{\mathbf{x}} \times \mathbf{R}$ with a controllability region $\Omega_{\mathbf{x}}$.

Remark 4. Taking into account [33] and the response bounds of the rigid-body states, we know that Assumption 2 holds.

To avoid the complex design process of backstepping, the following model transformation is made.

Step 1. Define $z_{1}=x_{1}=\gamma$ and $z_{2}=\dot{z}_{1}=f_{1}\left(x_{1}, x_{2}\right)$. Considering (37), the time derivative of $z_{2}$ is given by the following:

$$
\begin{aligned}
\dot{z}_{2} & =\frac{\partial f_{1}\left(x_{1}, x_{2}\right)}{\partial x_{1}} \dot{x}_{1}+\frac{\partial f_{1}\left(x_{1}, x_{2}\right)}{\partial x_{2}} \dot{x}_{2} \\
& =\frac{\partial f_{1}\left(x_{1}, x_{2}\right)}{\partial x_{1}} f_{1}\left(x_{1}, x_{2}\right)+\frac{\partial f_{1}\left(x_{1}, x_{2}\right)}{\partial x_{2}} x_{3} \\
& \triangleq f_{h 1}(\mathbf{x}) .
\end{aligned}
$$

Step 2. Define $z_{3}=\dot{z}_{2}=f_{h 1}(\mathbf{x})$. Invoking (37), the time derivative of $z_{3}$ is derived as follows:

$$
\begin{aligned}
\dot{z}_{3} & =\frac{\partial f_{h 1}(\mathbf{x})}{\partial x_{1}} \dot{x}_{1}+\frac{\partial f_{h 1}(\mathbf{x})}{\partial x_{2}} \dot{x}_{2}+\frac{\partial f_{h 1}(\mathbf{x})}{\partial x_{3}} \dot{x}_{3} \\
& =\frac{\partial f_{h 1}(\mathbf{x})}{\partial x_{1}} f_{1}\left(x_{1}, x_{2}\right)+\frac{\partial f_{h 1}(\mathbf{x})}{\partial x_{2}} x_{3}+\frac{\partial f_{h 1}(\mathbf{x})}{\partial x_{3}} f_{3}\left(\mathbf{x}, \delta_{\mathrm{e}}\right) \\
& \triangleq f_{h 2}\left(\mathbf{x}, \delta_{\mathrm{e}}\right) .
\end{aligned}
$$

As a result, a pure feedback model with a nonaffine formulation is obtained:

$$
\begin{aligned}
& \dot{z}_{1}=z_{2}, \\
& \dot{z}_{2}=z_{3}, \\
& \dot{z}_{3}=f_{h 2}\left(\mathbf{x}, \delta_{\mathrm{e}}\right),
\end{aligned}
$$

where $f_{h 2}\left(\mathbf{x}, \delta_{\mathrm{e}}\right)$ is an unknown continuous function.

Remark 5. From (37), (38), (39), and (40), we obtain that

$$
\begin{aligned}
\frac{\partial f_{h 2}\left(\mathbf{x}, \delta_{\mathrm{e}}\right)}{\partial \delta_{\mathrm{e}}} & =\frac{\partial f_{h 1}(\mathbf{x})}{\partial x_{3}} \frac{\partial f_{3}\left(\mathbf{x}, \delta_{\mathrm{e}}\right)}{\partial \delta_{\mathrm{e}}} \\
& =\frac{\partial f_{1}\left(x_{1}, x_{2}\right)}{\partial x_{2}} \frac{\partial f_{3}\left(\mathbf{x}, \delta_{\mathrm{e}}\right)}{\partial \delta_{\mathrm{e}}}>0 .
\end{aligned}
$$

Remark 6. Compared with (37), the expression of (41) is simpler. On the basis of (41), the complicated design process of backstepping can be eliminated.

Define flight-path angle tracking error $e_{0}$ and error function $E$ as follows:

$$
\begin{aligned}
& e_{0}=\gamma-\gamma_{d}=z_{1}-\gamma_{d}, \\
& E=\left(\frac{d}{d t}+\mu\right)^{3} \int_{0}^{t} e_{0} d \tau=\ddot{e}_{0}+3 \mu \dot{e}_{0}+3 \mu^{2} e_{0}+\mu^{3} \int_{0}^{t} e_{0} d \tau,
\end{aligned}
$$

where $\mu$ is a positive constant to be chosen. Moreover, because the polynomial $(s+\mu)^{3}$ is Hurwitz, the boundedness of $e_{0}$ can be guaranteed if $E$ is bounded.

Considering (41), the first three order time derivatives of $e_{0}$ are derived as follows:

$$
\begin{aligned}
& \dot{e}_{0}=\dot{z}_{1}-\dot{\gamma}_{d}=z_{2}-\dot{\gamma}_{d}, \\
& \ddot{e}_{0}=\dot{z}_{2}-\ddot{\gamma}_{d}=z_{3}-\ddot{\gamma}_{d}, \\
& \dddot{e}_{0}=\dot{z}_{3}-\dddot{\gamma}_{d}=k_{h} \delta_{\mathrm{e}}+F_{h}\left(\mathbf{x}, \delta_{\mathrm{e}}\right)-\dddot{\gamma}_{d},
\end{aligned}
$$

where $k_{h}>0$ is a design parameter; $F_{h}\left(\mathbf{x}, \delta_{\mathrm{e}}\right)=f_{h 2}\left(\mathbf{x}, \delta_{\mathrm{e}}\right)-$ $k_{h} \delta_{\mathrm{e}}$ is a completely unknown function. 
Thus, the time derivative of $E$ is given as follows:

$$
\begin{aligned}
\dot{E} & =\dddot{e}_{0}+3 \mu \ddot{e}_{0}+3 \mu^{2} \dot{e}_{0}+\mu^{3} e_{0} \\
& =k_{h} \delta_{\mathrm{e}}+F_{h}\left(\mathbf{x}, \delta_{\mathrm{e}}\right)-\dddot{\gamma}_{d}+3 \mu \ddot{e}_{0}+3 \mu^{2} \dot{e}_{0}+\mu^{3} e_{0} .
\end{aligned}
$$

Note that the newly defined states $z_{2}$ and $z_{3}$ are unknown. From the above model transformation process, we have that $z_{2}=\dot{\gamma}$ and $z_{3}=\ddot{\gamma}$. Thus, let $\gamma$ pass through the HTD presented in Section 2.3. We can get the estimations of $z_{2}$ and $z_{3}$, denoted by $\widehat{z}_{2}$ and $\widehat{z}_{3}$, respectively. Meanwhile, let $\gamma_{d}$ be the input signal of the HTD. We can obtain the estimations of $\dot{\gamma}_{d}, \ddot{\gamma}_{d}$, and $\dddot{\gamma}_{d}$, represented by $\dot{\hat{\gamma}}_{d}, \ddot{\hat{\gamma}}_{d}$, and $\dddot{\widehat{\gamma}}_{d}$, respectively. The relative estimation errors are defined as follows:

$$
\begin{aligned}
& s_{1}=\widehat{z}_{2}-z_{2}, \\
& s_{2}=\widehat{z}_{3}-z_{3}, \\
& s_{3}=\hat{\widehat{\gamma}}_{d}-\dot{\gamma}_{d}, \\
& s_{4}=\ddot{\hat{\gamma}}_{d}-\ddot{\gamma}_{d}, \\
& s_{5}=\dddot{\widehat{\gamma}}_{d}-\dddot{\gamma}_{d} .
\end{aligned}
$$

By Remark 1, it is concluded that there exist positive constants $\bar{s}_{i}(i=1-5)$ such that $\bar{s}_{i} \geq\left|s_{i}\right|(i=1-5)$. Thus, (44) and (45) can be modified as follows:

$$
\begin{gathered}
\dot{\widehat{e}}_{0}=\widehat{z}_{2}-\dot{\hat{\gamma}}_{d}, \\
\ddot{\widehat{e}}_{0}=\widehat{z}_{3}-\ddot{\widehat{\gamma}}_{d}, \\
\dddot{\widehat{e}}_{0}=k_{h} \delta_{e}+F_{h}\left(\mathbf{x}, \delta_{e}\right)-\dddot{\widehat{\gamma}}_{d}, \\
\dot{\widehat{E}}=k_{h} \delta_{e}+F_{h}\left(\mathbf{x}, \delta_{e}\right)-\dddot{\widehat{\gamma}}_{d}+3 \mu \ddot{\widehat{e}}_{0}+3 \mu^{2} \dot{\widehat{e}}_{0}+\mu^{3} e_{0} .
\end{gathered}
$$

The control law is selected as follows:

$$
\delta_{\mathrm{e}}=k_{h}^{-1}\left(\delta_{\mathrm{e} 0}-\delta_{\mathrm{e} 1}\right)
$$

where $\delta_{\mathrm{e} 0}=-k_{h 1} \widehat{E}+\dddot{\widehat{\gamma}}_{d}-3 \mu \ddot{\widehat{e}}_{0}-3 \mu^{2} \dot{\widehat{e}}_{0}-\mu^{3} e_{0} ; k_{h}>0, k_{h 1}>0$ are design parameters; $\delta_{\mathrm{e} 1}$ is a neural control signal devised to cancel $F_{h}\left(\mathbf{x}, \delta_{\mathrm{e}}\right)$ by applying an RBFNN.

Considering Lemma 1 , we know that there exists a $\delta_{\mathrm{e} 1}^{*}$ such that

$$
H_{2}\left(\mathbf{x}, \delta_{\mathrm{e} 0}, \delta_{\mathrm{e} 1}^{*}\right) \triangleq F_{h}\left(\mathbf{x}, k_{h}^{-1}\left(\delta_{\mathrm{e} 0}-\delta_{\mathrm{e} 1}^{*}\right)\right)-\delta_{\mathrm{e} 1}^{*}=0 .
$$

Theorem 3. Define

$$
k_{h}>\frac{1}{2} \frac{\partial f_{h 2}\left(\mathbf{x}, \delta_{\mathrm{e}}\right)}{\partial \delta_{\mathrm{e}}} .
$$

Then, there exist a set $\Omega_{\mathbf{x}} \subset \mathbf{R}^{3}$ and a unique $\delta_{\mathrm{e} 1}^{*}$ that is a function of $\boldsymbol{x}$ and $\delta_{\mathrm{e}}$ such that $\delta_{\mathrm{e} 1}^{*}\left(\mathbf{x}, \delta_{\mathrm{e} 0}\right)$ satisfies (50) for all $\left(\mathbf{x}, \delta_{\mathrm{e} 0}\right) \in \Omega_{\mathbf{x}} \times \mathbf{R}$.
Proof. The sufficient condition for the existence of $\delta_{\mathrm{el}}^{*}$ is that the following inequality holds $[37,38]$ :

$$
\left|\frac{\partial F_{h}\left(\mathbf{x}, \delta_{\mathrm{e}}\right)}{\partial \delta_{\mathrm{e} 1}^{*}}\right|<1 .
$$

Considering (42), (49), and (51), it follows that

$$
\begin{aligned}
\left|\frac{\partial F_{h}\left(\mathbf{x}, \delta_{\mathrm{e}}\right)}{\partial \delta_{\mathrm{e} 1}^{*}}\right| & =\left|\frac{\partial\left[f_{h 2}\left(\mathbf{x}, \delta_{\mathrm{e}}\right)-k_{h} \delta_{\mathrm{e}}\right]}{\partial \delta_{\mathrm{e} 1}^{*}}\right| \\
& =\left|\frac{\partial\left[f_{h 2}\left(\mathbf{x}, \delta_{\mathrm{e}}\right)-k_{h} \delta_{\mathrm{e}}\right]}{\partial \delta_{\mathrm{e}}} \frac{\partial \delta_{\mathrm{e}}}{\partial \delta_{\mathrm{e} 1}^{*}}\right| \\
& =\left|\left[\frac{\partial f_{h 2}\left(\mathbf{x}, \delta_{\mathrm{e}}\right)}{\partial \delta_{\mathrm{e}}}-k_{h}\right] \frac{1}{k_{h}}\right| \\
& =\left|\frac{1}{k_{h}} \frac{\partial f_{h 2}\left(\mathbf{x}, \delta_{\mathrm{e}}\right)}{\partial \delta_{\mathrm{e}}}-1\right|<1 .
\end{aligned}
$$

Thereby, $\delta_{\mathrm{e} 1}^{*}$ exists.

Furthermore,

$$
\begin{aligned}
\frac{\partial}{\partial \delta_{\mathrm{e} 1}^{*} H_{2}\left(\mathbf{x}, \delta_{\mathrm{e} 0}, \delta_{\mathrm{e} 1}^{*}\right)} & =\frac{\partial}{\partial \delta_{\mathrm{e} 1}^{*}}\left[F_{h}\left(\mathbf{x}, \delta_{\mathrm{e}}^{*}\right)-\delta_{\mathrm{e} 1}^{*}\right] \\
& =\frac{\partial}{\partial \delta_{\mathrm{e} 1}^{*}}\left[f_{h 2}\left(\mathbf{x}, \delta_{\mathrm{e}}^{*}\right)-k_{h} \delta_{\mathrm{e}}^{*}\right]-1 \\
& =\frac{\partial}{\partial \delta_{\mathrm{e}}^{*}}\left[f_{h 2}\left(\mathbf{x}, \delta_{\mathrm{e}}^{*}\right)-k_{h} \delta_{\mathrm{e}}^{*}\right] \frac{\partial \delta_{\mathrm{e}}^{*}}{\partial \delta_{\mathrm{e} 1}^{*}}-1 \\
& =\left[\frac{\partial f_{h 2}\left(\mathbf{x}, \delta_{\mathrm{e}}^{*}\right)}{\partial \delta_{\mathrm{e}}^{*}}-k_{h}\right]\left(-\frac{1}{k_{h}}\right)-1 \\
& =-\frac{1}{k_{h}} \frac{\partial f_{h 2}\left(\mathbf{x}, \delta_{\mathrm{e}}^{*}\right)}{\partial \delta_{\mathrm{e}}^{*}},
\end{aligned}
$$

with $\delta_{\mathrm{e}}^{*}=k_{h}^{-1}\left(\delta_{\mathrm{e} 0}-\delta_{\mathrm{e} 1}^{*}\right)$. Obviously, $\partial H_{2}\left(\mathbf{x}, \delta_{\mathrm{e} 0}, \delta_{\mathrm{e} 1}^{*}\right) /\left(\partial \delta_{\mathrm{e} 1}^{*}\right)$ is nonsingular. Hence, Theorem 3 is satisfied. The proof is completed.

It is observed from Theorem 3 and Lemma 1 that $\delta_{e}$ can be treated as a function of $\mathbf{x}$ and $\delta_{\mathrm{e} 0}$ because of $\delta_{\mathrm{e}}=k_{h}^{-1}$ $\left(\delta_{\mathrm{e} 0}-\delta_{\mathrm{e} 1}\right)$. Hence, $F_{h}\left(\mathbf{x}, \delta_{\mathrm{e}}\right)=f_{h 2}\left(\mathbf{x}, \delta_{\mathrm{e}}\right)-k_{h} \delta_{\mathrm{e}}$ can also be viewed as a function of $\mathbf{x}$ and $\delta_{\mathrm{e} 0}$.

Define $\mathbf{X}_{2}=\left[\mathbf{x}^{T}, \delta_{\mathrm{e} 0}\right]^{T} \in \mathbf{R}^{4}$ as an input vector. Then, we employ an RBFNN to approximate $F_{h}\left(\mathbf{x}, \delta_{\mathrm{e}}\right)$.

$$
F_{h}\left(\mathbf{x}, \delta_{\mathrm{e}}\right)=\mathbf{W}_{2}^{* T} \mathbf{h}_{2}\left(\mathbf{X}_{2}\right)+\iota_{2}, \quad\left|\iota_{2}\right| \leq \iota_{2 M},
$$

where $\mathbf{W}_{2}^{*}=\left[w_{21}^{*}, w_{22}^{*}, \ldots, w_{2 p_{2}}^{*}\right]^{T} \in \mathbf{R}^{p_{2}}$ means an ideal weight vector; $\iota_{2}$ and $\iota_{2 \mathrm{M}}$ represent the approximation error and its upper bound, respectively; $\mathbf{h}_{2}\left(\mathbf{X}_{2}\right)=\left[h_{21}\left(\mathbf{X}_{2}\right), h_{22}\left(\mathbf{X}_{2}\right), \ldots\right.$, $\left.h_{2 p_{2}}\left(\mathbf{X}_{2}\right)\right]^{T} \in \mathbf{R}^{p_{2}}, h_{2 j}\left(\mathbf{X}_{2}\right)\left(j=1,2, \ldots, p_{2}\right)$ have similar formulations to (11).

$\delta_{\mathrm{e} 1}$ is chosen as follows:

$$
\delta_{\mathrm{el}}=\frac{1}{2} \widehat{E} \widehat{\varphi}_{2} \mathbf{h}_{2}^{T}\left(\mathbf{X}_{2}\right) \mathbf{h}_{2}\left(\mathbf{X}_{2}\right),
$$


where $\varphi_{2}=\left\|\mathbf{W}_{2}^{*}\right\|^{2}, \widehat{\varphi}_{2}$ is the estimation of $\varphi_{2}$ with the following adaptive law:

$$
\dot{\hat{\varphi}}_{2}=\frac{\lambda_{2}}{2} \widehat{E}^{2} \mathbf{h}_{2}^{T}\left(\mathbf{X}_{2}\right) \mathbf{h}_{2}\left(\mathbf{X}_{2}\right)-2 \widehat{\varphi}_{2},
$$

with $\lambda_{2}>0$.

Theorem 4. Consider the closed-loop system consisting of plant (41) under Assumption 2 with controller (50) and adaptive law (58). Then all the signals involved are semi-globally uniformly ultimately bounded.

Proof. Define

$$
\widetilde{\varphi}_{2}=\widehat{\varphi}_{2}-\varphi_{2}
$$

Substituting (49), (55), and (56) into (48), we have

$$
\dot{\hat{E}}=-k_{h 1} \widehat{E}-\frac{1}{2} \widehat{E} \widehat{\varphi}_{2} \mathbf{h}_{2}^{T}\left(\mathbf{X}_{2}\right) \mathbf{h}_{2}\left(\mathbf{X}_{2}\right)+\mathbf{W}_{2}^{* T} \mathbf{h}_{2}\left(\mathbf{X}_{2}\right)+\iota_{2} .
$$

Design the following Lyapunov function candidate:

$$
W_{h}=\frac{1}{2} \widehat{E}^{2}+\frac{\widetilde{\varphi}_{2}^{2}}{2 \lambda_{2}} .
$$

Differentiating along (60) with respect to time and substituting (57), (58), and (59), we obtain

$$
\begin{aligned}
\dot{W}_{h}= & \widehat{E} \dot{\hat{E}}+\frac{\widetilde{\varphi}_{2} \dot{\hat{\varphi}}_{2}}{\lambda_{2}} \\
= & -k_{h 1} \widehat{E}^{2}-\frac{1}{2} \widehat{E}^{2} \varphi_{2} \mathbf{h}_{2}^{T}\left(\mathbf{X}_{2}\right) \mathbf{h}_{2}\left(\mathbf{X}_{2}\right)+\widehat{E} \mathbf{W}_{2}^{* T} \mathbf{h}_{2}\left(\mathbf{X}_{2}\right) \\
& +\iota_{2} \widehat{E}-\frac{2 \widetilde{\varphi}_{2} \widehat{\varphi}_{2}}{\lambda_{2}}
\end{aligned}
$$

Notice that

$$
\begin{aligned}
\widehat{E} \mathbf{W}_{2}^{* T} \mathbf{h}_{2}\left(\mathbf{X}_{2}\right) & \leq \frac{\widehat{E}^{2}}{2}\left\|\mathbf{W}_{2}^{* T} \mathbf{h}_{2}\left(\mathbf{X}_{2}\right)\right\|^{2}+\frac{1}{2} \\
& =\frac{\widehat{E}^{2}}{2}\left\|\mathbf{W}_{2}^{*}\right\|^{2}\left\|\mathbf{h}_{2}\left(\mathbf{X}_{2}\right)\right\|^{2}+\frac{1}{2} \\
& =\frac{\widehat{E}^{2}}{2} \varphi_{2} \mathbf{h}_{2}^{T}\left(\mathbf{X}_{2}\right) \mathbf{h}_{2}\left(\mathbf{X}_{2}\right)+\frac{1}{2} \frac{2 \widetilde{\varphi}_{2} \widehat{\varphi}_{2}}{\lambda_{2}} \\
& \geq \frac{\widetilde{\varphi}_{2}^{2}}{\lambda_{2}}-\frac{\varphi_{2}^{2}}{\lambda_{2}}, \\
\widehat{E} \iota_{2} & \leq \frac{1}{2} \widehat{E}^{2} \iota_{2 M}^{2}+\frac{1}{2} .
\end{aligned}
$$

Thus, the following inequality is satisfied:

$$
\dot{W}_{h}=-\left(k_{h 1}-\frac{1}{2} \iota_{2 M}^{2}\right) \widehat{E}^{2}-\frac{1}{\lambda_{2}} \widetilde{\varphi}_{2}^{2}+1+\frac{\varphi_{2}^{2}}{\lambda_{2}} .
$$

TABLE 1: Initial trim conditions.

\begin{tabular}{lcc}
\hline Item & Value & Units \\
\hline$V$ & 7700 & $\mathrm{ft} / \mathrm{s}$ \\
$h$ & 85,000 & $\mathrm{ft}$ \\
$\gamma$ & 0 & $\mathrm{deg}$ \\
$\theta$ & 1.6325 & $\mathrm{deg}$ \\
$Q$ & 0 & $\mathrm{deg} / \mathrm{s}$ \\
$\eta_{1}$ & 0.9700 & - \\
$\dot{\eta}_{1}$ & 0 & - \\
$\eta_{2}$ & 0.7967 & - \\
$\dot{\eta}_{2}$ & 0 & - \\
\hline
\end{tabular}

Let $k_{h 1}>\iota_{2 M}^{2} / 2$ and define the following compact sets:

$$
\begin{aligned}
& \Omega_{\widehat{E}}=\left\{\widehat{E}|| \widehat{E} \mid \leq \sqrt{\frac{1+\left(\varphi_{2}^{2} / \lambda_{2}\right)}{k_{h 1}-(1 / 2) \iota_{2 M}^{2}}}\right\}, \\
& \Omega_{\tilde{\varphi}_{2}}=\left\{\widetilde{\varphi}_{2}\left|\widetilde{\varphi}_{2}\right| \leq \sqrt{\frac{1+\left(\varphi_{2}^{2} / \lambda_{2}\right)}{\left(1 / \lambda_{2}\right)}}\right\} .
\end{aligned}
$$

It is clear that $\dot{W}_{h}$ will be negative if $\widehat{E} \notin \Omega_{\widehat{E}}$ or $\widetilde{\varphi}_{2} \notin \Omega_{\tilde{\varphi}_{2}}$. Therefore, $\widehat{E}$ and $\widetilde{\varphi}_{2}$ are semiglobally uniformly ultimately bounded. The proof is completed.

Remark 7. From (43), (44), (45), (46), (47), and (48), we get that

$$
\begin{aligned}
\widehat{E} & =\ddot{\widehat{e}}_{0}+3 \mu \dot{\hat{e}}_{0}+3 \mu^{2} e_{0}+\mu^{3} \int_{0}^{t} e_{0} \mathrm{~d} \tau \\
& =\ddot{e}_{0}+s_{2}-s_{4}+3 \mu\left(\dot{e}_{0}+s_{1}-s_{3}\right)+3 \mu^{2} e_{0}+\mu^{3} \int_{0}^{t} e_{0} \mathrm{~d} \tau \\
& =E+s_{2}-s_{4}+3 \mu\left(s_{1}-s_{3}\right) .
\end{aligned}
$$

It further follows that

$$
|E|=\left|\widehat{E}-s_{2}+s_{4}-3 \mu\left(s_{1}-s_{3}\right)\right| \leq|\widehat{E}|+\bar{s}_{2}+\bar{s}_{4}+3 \mu\left(\bar{s}_{1}+\bar{s}_{3}\right) .
$$

Thus, $E$ and $e_{0}$ are also bounded. That is, $\gamma$ can converge to $\gamma_{d}$.

Remark 8. Different from the affine control strategies [26$30]$, the proposed controller is derived from a nonaffine model such that it exhibits better practicability. Moreover, the prior information [30] that the unknown control gains approximated by NNs have to be strictly positive is not needed in this paper.

Remark 9. It is obvious that there is no need of the complicated design process of backstepping. Compared with $[26,30]$, the architecture of the developed controller is more concise. Meanwhile, the problem of "explosion of terms" is successfully eliminated. 


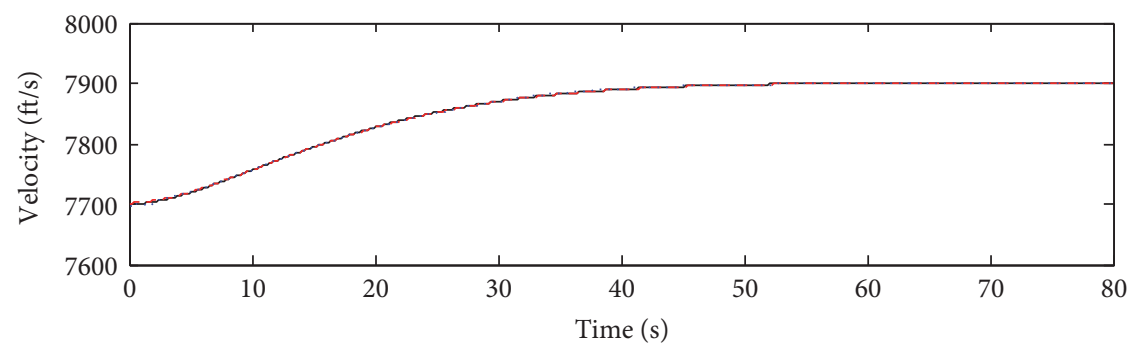

$-V_{\text {ref }}$

- - - The proposed controller Backstepping controller

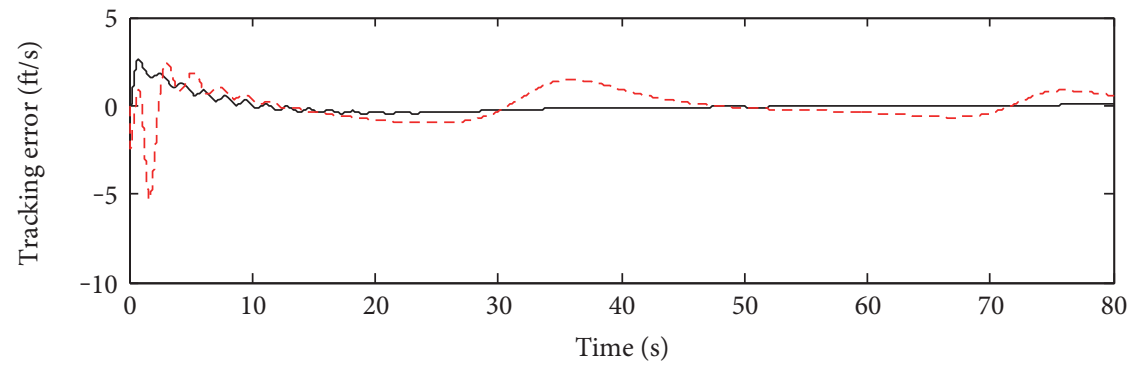

— The proposed controller

- - Backstepping controller

Figure 2: Velocity tracking performance.

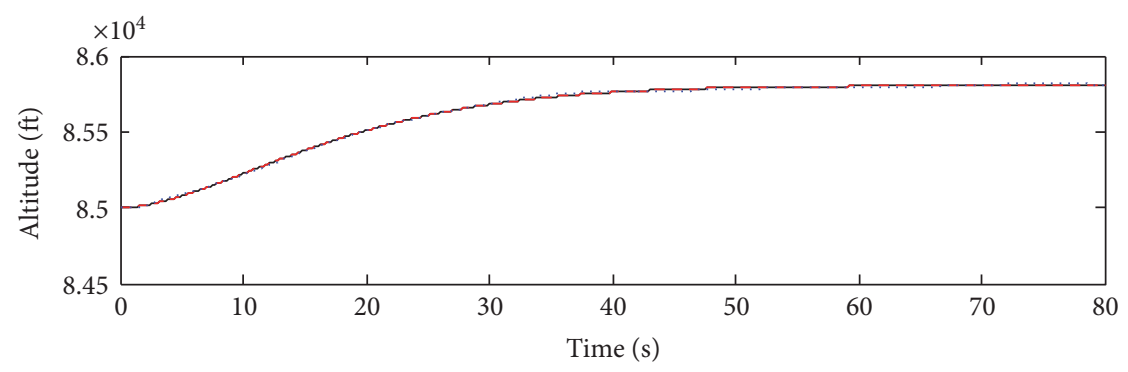

$-h_{\text {ref }}$

- - - The proposed controller

Backstepping controller

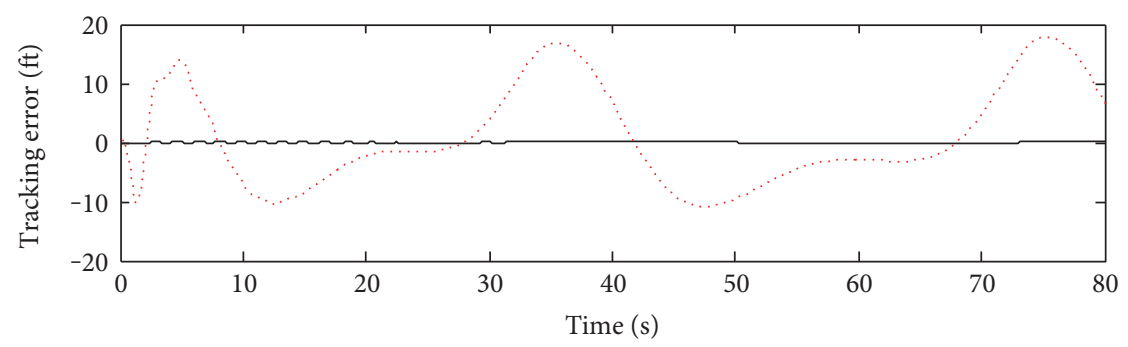

— The proposed controller Backstepping controller

FIGURE 3: Altitude tracking performance.

Remark 10. Throughout this paper, only two NNs are applied to approach the lumped uncertainties of both subsystems. Furthermore, by the merit of the MLP scheme, there are totally only two learning parameters (i.e., $\widehat{\varphi}_{1}$ and $\widehat{\varphi}_{2}$ ) required for neural approximation. In comparison with [30], a lower computational burden design is achieved in this study.

Remark 11. If the input vectors of RBFNNs are chosen as $\mathbf{X}_{1}=[V, \Phi]^{T}$ and $\mathbf{X}_{2}=\left[\mathbf{x}^{T}, \delta_{\mathrm{e}}\right]^{T}$, it will lead to a fixed-point 

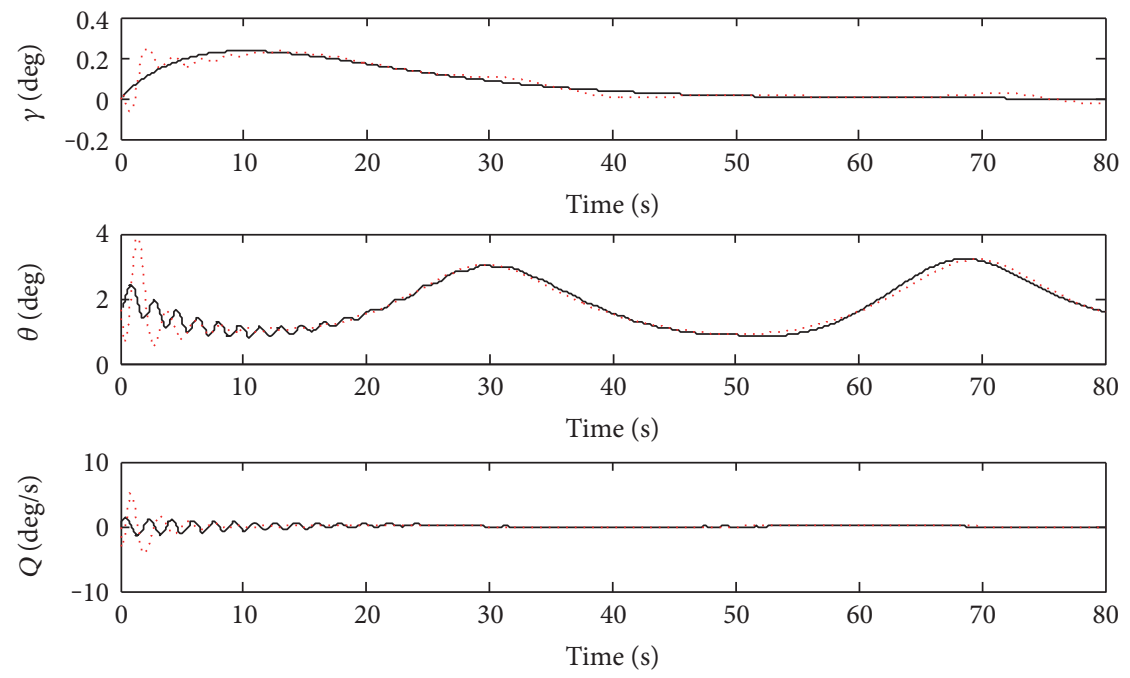

The proposed controller Backstepping controller

FIgURE 4: The attitude angles.
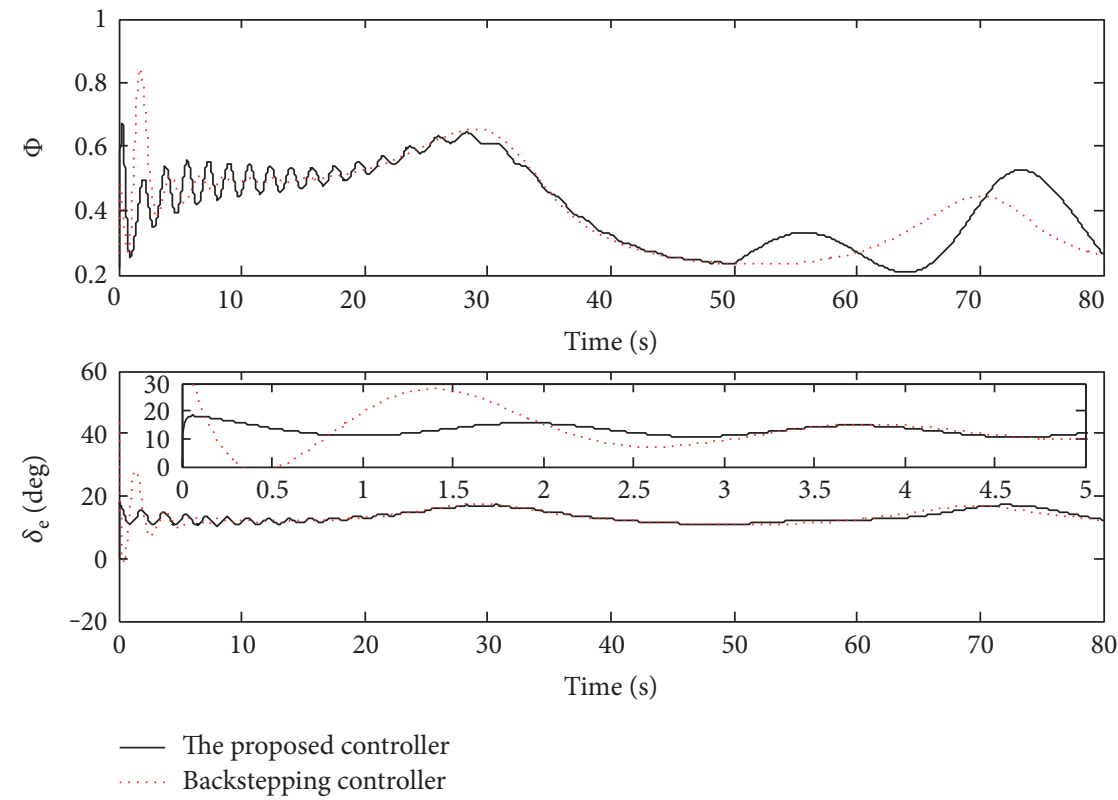

FIgURE 5: The control inputs.

problem, which will result in a computational burden. In this paper, such problem is avoided.

\section{Simulation Results}

In this section, we will make simulation studies to validate the tracking performance of the proposed control methodology. All the aerodynamic coefficients and parameters in (1), (2), (3), (4), (5), (6), and (7) are the same as [33]. The initial trim conditions of the vehicle are shown in Table 1 . The reference trajectories of velocity and altitude are smoothed via the following filter:

$$
\frac{0.1^{2}}{s^{2}+2 \times 0.9 \times 0.1 \times s+0.1^{2}} .
$$

The centers of RBFNNs are evenly spaced in $\mathbf{c}_{1} \in[7700$ $\mathrm{ft} / \mathrm{s}, 8700 \mathrm{ft} / \mathrm{s}] \times[-0.1,1], \mathbf{c}_{2} \in[-1 \mathrm{deg}, 1 \mathrm{deg}] \times[0 \mathrm{deg}, 5 \mathrm{deg}]$ $\times[-5 \mathrm{deg} / \mathrm{s}, 5 \mathrm{deg} / \mathrm{s}] \times[0 \mathrm{rad}, 0.35 \mathrm{rad}]$. The weight vectors are chosen as $\mathbf{b}_{1}=[33.33 \mathrm{ft} / \mathrm{s}, 0.0367]^{T}, \mathbf{b}_{2}=[0.0012 \mathrm{rad}$, $0.0029 \mathrm{rad}, 0.0058 \mathrm{rad} / \mathrm{s}, 0.0117 \mathrm{rad}]^{T}$. The node number is 

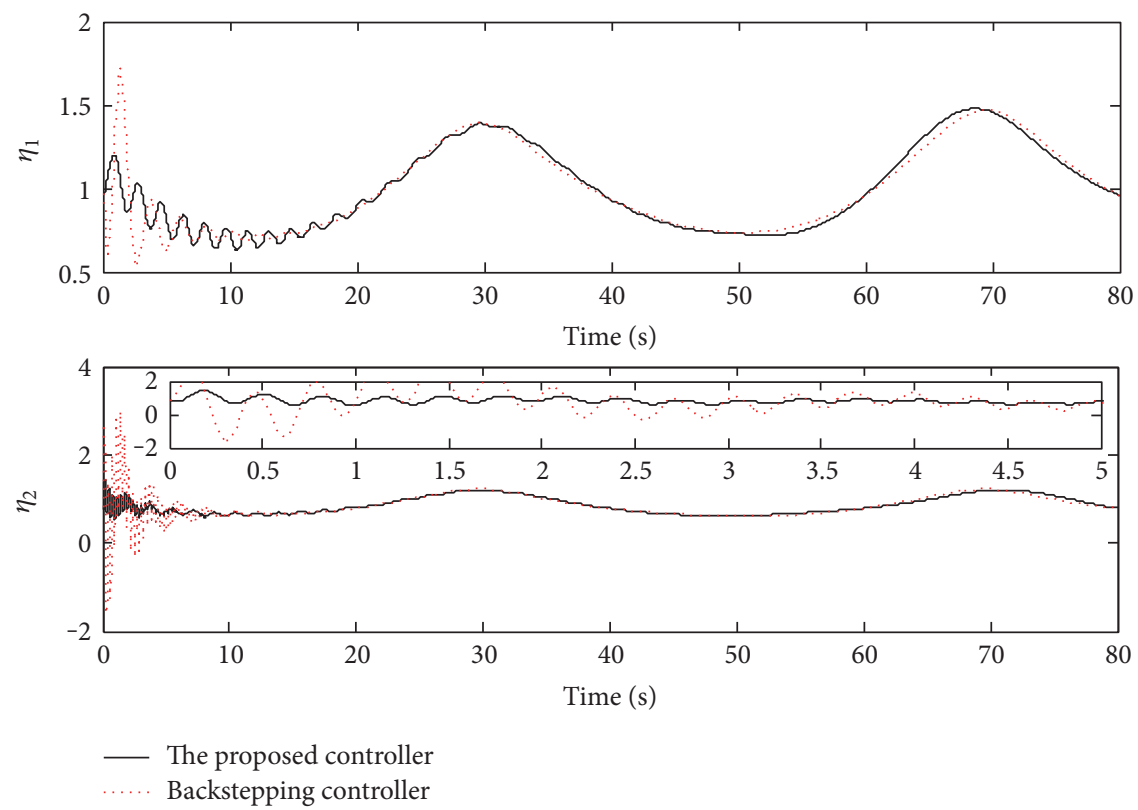

Figure 6: The flexible states.
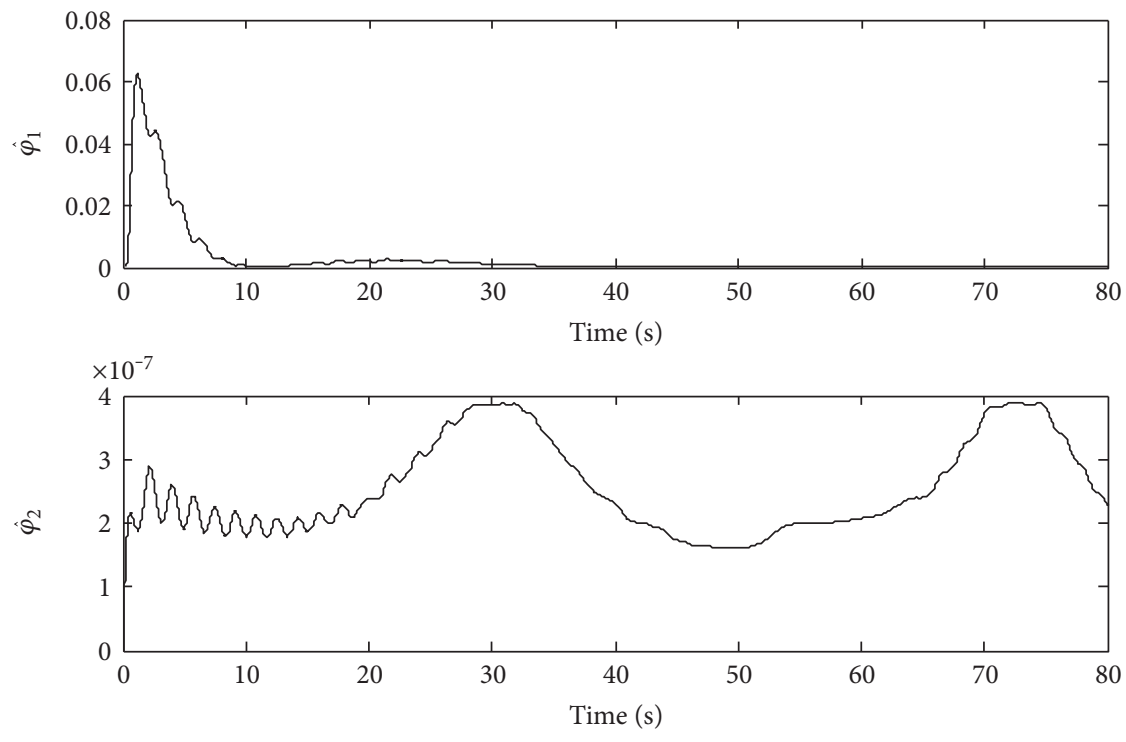

The proposed controller

FIgURE 7: The estimations of $\varphi_{1}$ and $\varphi_{2}$.

set as $p_{1}=p_{2}=20$. The design parameters are chosen as follows: $k_{V}=0.9, k_{V 1}=0.2, k_{V 2}=0.8, k_{\gamma}=2, k_{h}=0.9, k_{h 1}=50$, $\mu=7, \lambda_{1}=\lambda_{2}=0.05, R=0.05, a_{1}=a_{3}=0.5, a_{2}=a_{4}=0.1$. To show the superiority, the proposed controller is compared with a traditional backstepping control strategy presented in [19]. The following two cases are considered.

Case 1. In this case, the AHV is assumed to climb a maneuver from the initial trim conditions, listed in Table 1, to the final values $h=85800 \mathrm{ft}$ and $V=7900 \mathrm{ft} / \mathrm{s}$. To test the robustness of the designed controller, we define

$$
C=C_{0}[1+0.4 \sin (0.05 \pi t)]
$$

where $C$ means the value of uncertain coefficient and $C_{0}$ stands for the nominal value of $C$. With this definition, a parameter uncertainty up to $40 \%$ of the nominal value is considered.

The simulation results are shown in Figures 2, 3, 4, 5, 6, and 7. From Figures 2 and 3, it can be seen that the obtained velocity and altitude tracking performance is 

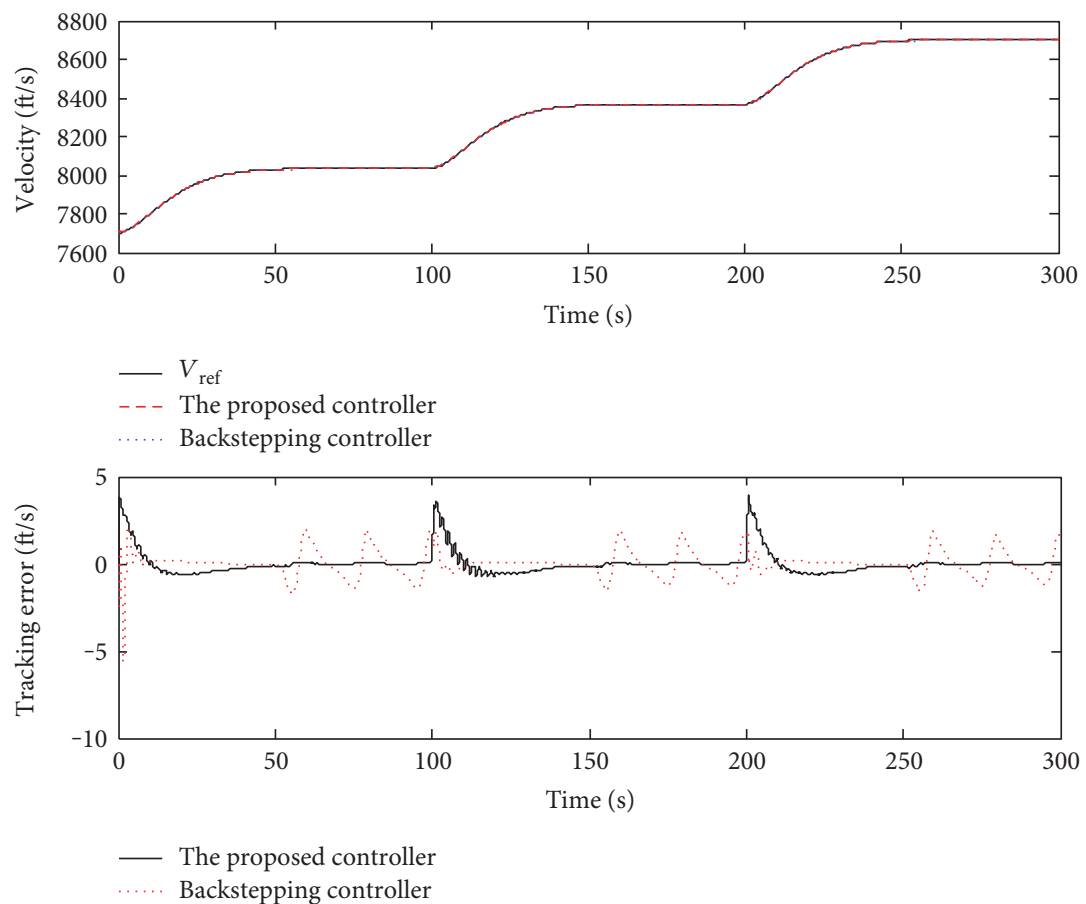

FIGURE 8: Velocity tracking and tracking error.
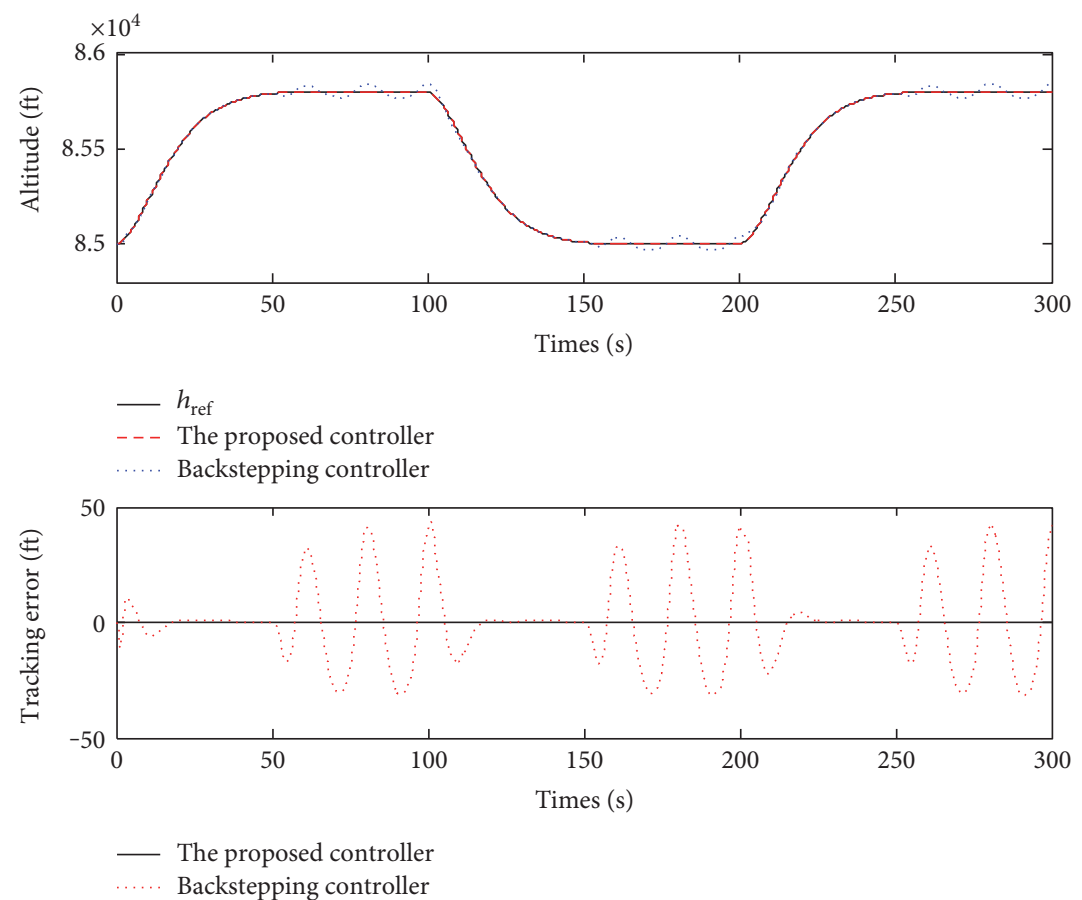

FIGURE 9: Altitude tracking and tracking error.

better than the one provided by the backstepping controller. Meanwhile, the attitude angles, the control inputs, and the flexible states, achieved by both control schemes, are smooth and without high-frequency chattering, as depicted in Figures 4, 5, and 6. Figure 7 reveals that the estimations of $\varphi_{1}$ and $\varphi_{2}$ are bounded.
Case 2. In this case, we take into consideration a more practical scenario. The altitude follows a square command with period $200 \mathrm{~s}$ and magnitude $800 \mathrm{ft}$. The velocity tracks the step command with $333.33 \mathrm{ft} / \mathrm{s}$ for every 100 seconds. The following condition of parametric uncertainty is considered. 

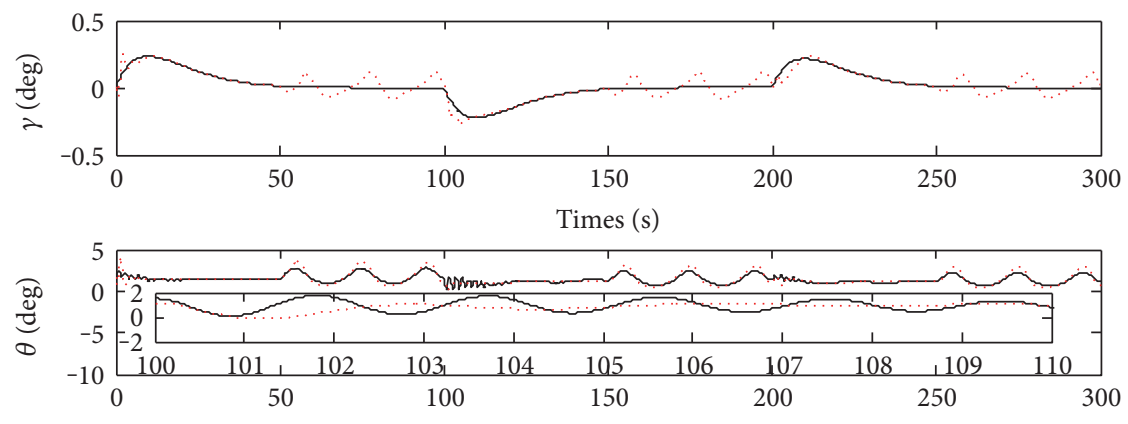

Times (s)

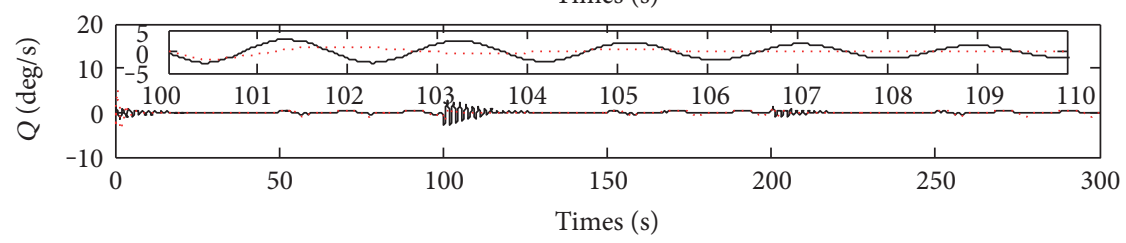

- The proposed controller Backstepping controller

Figure 10: The flight-path angle, pitch angle, and pitch rate.
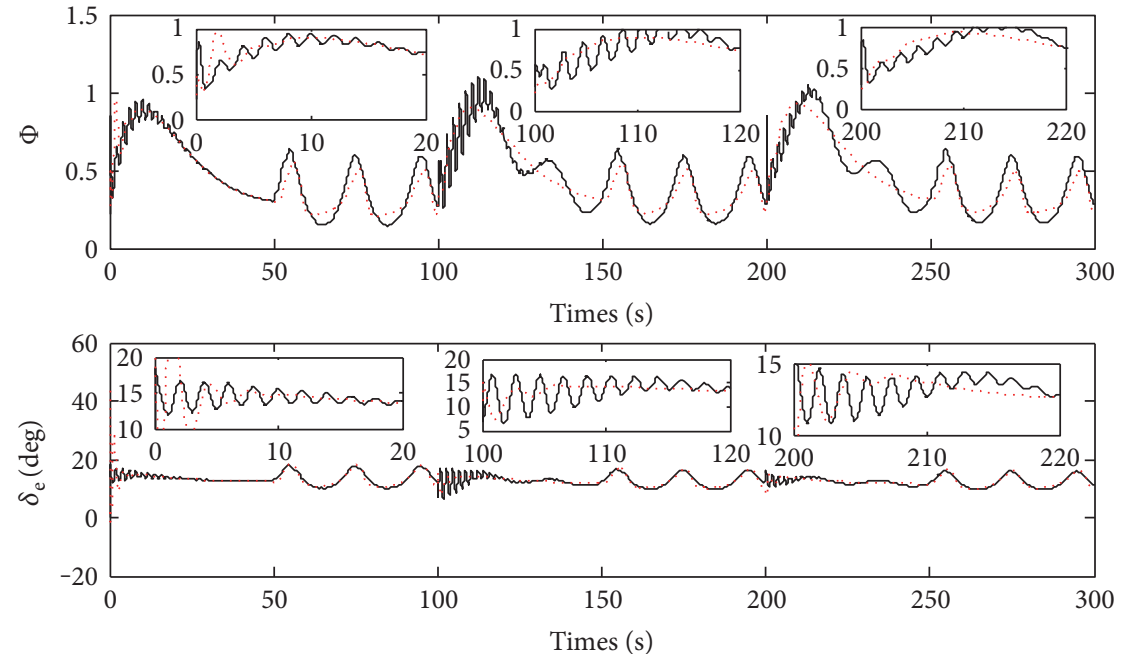

- The proposed controller Backstepping controller

FIgURE 11: The control inputs.

$$
C=\left\{\begin{array}{lc}
C_{0}, & 0 s \leq t<50 \mathrm{~s} \\
C_{0}[1+0.4 \sin (0.1 \pi t)], & 50 \mathrm{~s} \leq t<100 \mathrm{~s} \\
C_{0}, & 100 \mathrm{~s} \leq t<150 \mathrm{~s} \\
C_{0}[1+0.4 \sin (0.1 \pi t)], & 150 \mathrm{~s} \leq t<200 \mathrm{~s} \\
C_{0}, & 200 \mathrm{~s} \leq t<250 \mathrm{~s} \\
C_{0}[1+0.4 \sin (0.1 \pi t)], & 250 \mathrm{~s} \leq t \leq 300 \mathrm{~s}
\end{array}\right.
$$

The obtained simulation results, presented in Figures 8, $9,10,11,12$, and 13, indicate that the exploited control methodology can provide stable tracking of velocity and altitude commands in the presence of severe uncertainties.
Moreover, it is observed from Figures 8 and 9 that compared with backstepping control, much smaller tracking errors are achieved by the explored control algorithm when the model parameters are uncertain. The attitude angles, the control inputs, and the flexible states, shown in Figures 10, 11, and 12, are even and can converge to stable values. Finally, the boundedness of $\widehat{\varphi}_{1}$ and $\widehat{\varphi}_{2}$ is presented in Figure 13.

\section{Conclusions}

This paper investigates the design of a concise neural controller for an AHV with parametric uncertainties. The vehicle 

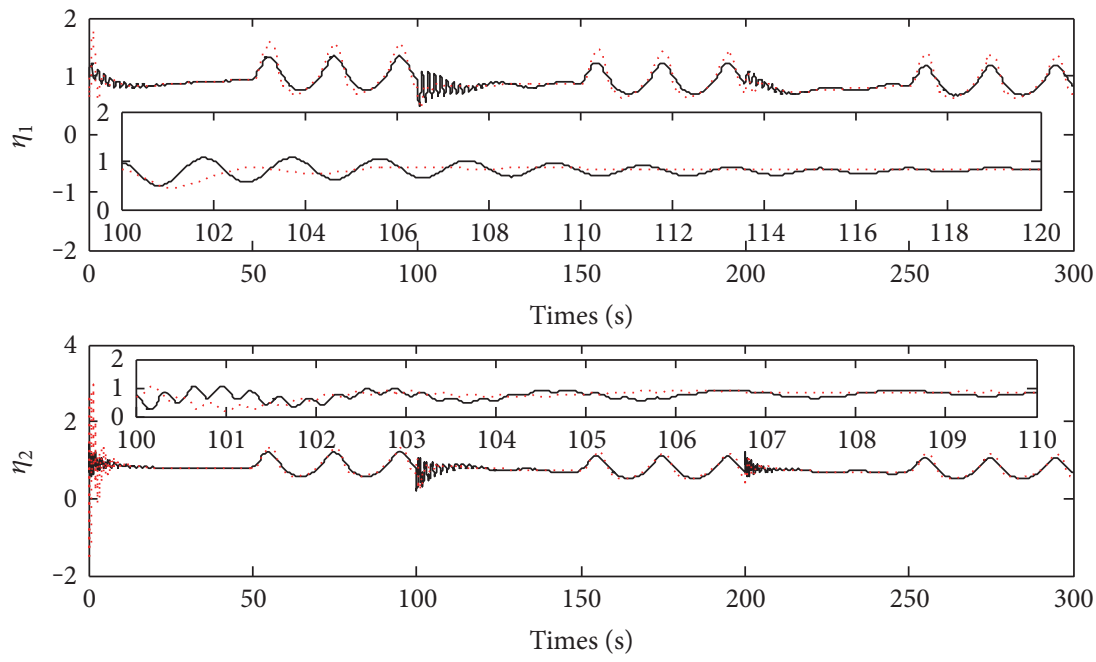

- The proposed controller Backstepping controller

FIGURE 12: The flexible states.
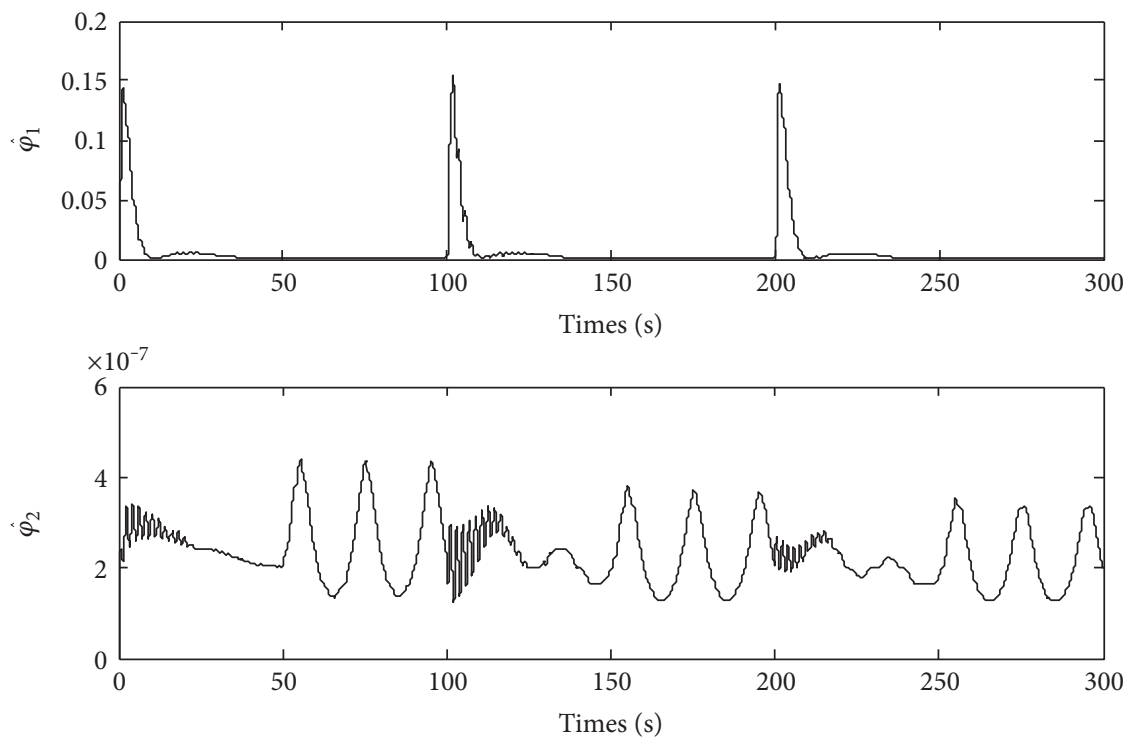

_ The proposed controller

FIGURE 13: The estimations of $\varphi_{1}$ and $\varphi_{2}$.

dynamics is decomposed into the respective velocity and altitude subsystems that are controlled separately. For both subsystems, simplified neural controllers are devised directly employing nonaffine models. Specially, a novel control scheme with a concise structure is proposed for the altitude dynamics without utilizing backstepping. Throughout this paper, only two NNs are applied and only two learning parameters are required for neural approximation. It is proved that all the closed-loop system signals are semiglobally uniformly ultimately bounded. Finally, the superiority of the design in command tracking over the traditional backstepping control is well validated by simulation results in the presence of model uncertainties.

\section{Nomenclature}

$\begin{array}{ll}m: & \text { Vehicle mass } \\ \rho: & \text { Density of air } \\ \bar{q}: & \text { Dynamic pressure } \\ S: & \text { Reference area } \\ h: & \text { Altitude } \\ V: & \text { Velocity } \\ \gamma: & \text { Flight-path angle } \\ \theta: & \text { Pitch angle } \\ \alpha: & \text { Angle of attack }(\alpha=\theta-\gamma) \\ Q: & \text { Pitch rate } \\ T: & \text { Thrust }\end{array}$




$\begin{array}{ll}D: & \text { Drag } \\ L: & \text { Lift } \\ M: & \text { Pitching moment } \\ I_{y y}: & \text { Moment of inertia } \\ \bar{c}: & \text { Aerodynamic chord } \\ z_{T}: & \text { Thrust moment arm } \\ \Phi: & \text { Fuel equivalence ratio } \\ \delta_{\mathrm{e}}: & \text { Elevator angular deflection } \\ N_{i}: & \text { ith generalized force } \\ N_{i}^{\alpha_{j}}: & \text { jth order contribution of } \alpha \text { to } N_{i} \\ N_{i}^{0}: & \text { Constant term in } N_{i} \\ N_{2}^{\delta_{\mathrm{e}}}: & \text { Contribution of } \delta_{\mathrm{e}} \text { to } N_{2} \\ \beta_{i}(h, \bar{q}): & \text { ith trust fit parameter } \\ \eta_{i}: & \text { ith generalized elastic coordinate } \\ \zeta_{i}: & \text { Damping ratio for elastic mode } \eta_{i} \\ \omega_{i}: & \text { Natural frequency for elastic mode } \eta_{i} \\ C_{D}^{\alpha^{i}}: & \text { ith order coefficient of } \alpha \text { in } D \\ C_{D}^{\delta_{\mathrm{e}}^{i}}: & \text { ith order coefficient of } \delta_{\mathrm{e}} \text { in } D \\ C_{D}^{0}: & \text { Constant coefficient in } D \\ C_{L}^{\alpha^{i}}: & \text { ith order coefficient of } \alpha \text { in } L \\ C_{L}^{\delta_{\mathrm{e}}}: & \text { Coefficient of } \delta_{\mathrm{e}} \text { contribution in } L \\ C_{L}^{0}: & \text { Constant coefficient in } L \\ C_{M, \alpha}^{\alpha^{i}}: & \text { ith order coefficient of } \alpha \text { in } M \\ C_{M, \alpha}^{0}: & \text { Constant coefficient in } M \\ C_{T}^{\alpha^{i}}: & \text { ith order coefficient of } \alpha \text { in } T \\ C_{T}^{0}: & \text { Constant coefficient in } T \\ h_{0}: & \text { Nominal altitude for air density approximation } \\ \rho_{0}: & \text { Air density at the altitude } h_{0} \\ \widetilde{\psi}_{i}: & \text { Constrained beam coupling constant for } \eta_{i} \\ c_{\mathrm{e}}: & \text { Coefficient of } \delta_{\mathrm{e}} \text { in } M \\ 1 / h_{s}: & \text { Air density decay rate } \\ \mathbf{R}^{n}: & \text { Th-dimensional Euclidean space } \\ \mathbf{R}: & \text { The set of all real numbers } \\ \left\|_{\bullet}\right\|:: & \text { The } 2 \text {-norm of a vector } \\ \mid \bullet: & \text { The absolute value of a scalar. } \\ & \end{array}$

\section{Conflicts of Interest}

The authors declare that they have no conflicts of interest.

\section{Acknowledgments}

This work was supported by the National Natural Science Foundation of China (Grant nos. 61603410 and 61573374) and Young Talent Fund of University Association for Science and Technology in Shaanxi, China (Grant no. 20170107).

\section{References}

[1] L. Fiorentini, A. Serrani, M. A. Bolender, and D. B. Doman, "Nonlinear robust adaptive control of flexible air-breathing hypersonic vehicles," Journal of Guidance, Control, and Dynamics, vol. 32, no. 2, pp. 401-416, 2009.

[2] C. Mu, Z. Ni, C. Sun, and H. He, "Air-breathing hypersonic vehicle tracking control based on adaptive dynamic programming," IEEE Transactions on Neural Networks and Learning Systems, vol. 28, no. 3, pp. 584-598, 2017.
[3] X. W. Bu, X. Y. Wu, J. Q. Huang, and D. Z. Wei, “A guaranteed transient performance-based adaptive neural control scheme with low-complexity computation for flexible air-breathing hypersonic vehicles," Nonlinear Dynamics, vol. 84, no. 4, pp. 2175-2194, 2016.

[4] C. Mu, C. Sun, and W. Xu, "Fast sliding mode control on air-breathing hypersonic vehicles with transient response analysis," Proceedings of the Institution of Mechanical Engineers, Part I: Journal of Systems and Control Engineering, vol. 230, no. 1, pp. 23-34, 2015.

[5] X. W. Bu, X. Y. Wu, J. Q. Huang, and D. Z. Wei, "Robust estimation-free prescribed performance back-stepping control of air-breathing hypersonic vehicles without affine models," International Journal of Control, vol. 89, no. 11, pp. 21852200, 2016.

[6] H. B. Sun, S. H. Li, and C. Y. Sun, "Finite time integral sliding mode control of hypersonic vehicles," Nonlinear Dynamics, vol. 73, no. 1-2, pp. 229-244, 2013.

[7] X. W. Bu, X. Y. Wu, J. Q. Huang, Z. Ma, and R. Zhang, "Minimal-learning-parameter based simplified adaptive neural back-stepping control of flexible air-breathing hypersonic vehicles without virtual controllers," Neurocomputing, vol. 175, pp. 816-825, 2016.

[8] J. M. Wang, Y. J. Wu, and X. M. Dong, "Recursive terminal sliding mode control for hypersonic flight vehicle with sliding mode disturbance observer," Nonlinear Dynamics, vol. 81, no. 3, pp. 1489-1510, 2015.

[9] X. X. Hu, L. G. Wu, C. H. Hu, and H. J. Gao, “Adaptive sliding mode tracking control for a flexible air-breathing hypersonic vehicle," Journal of the Franklin Institute, vol. 349, no. 2, pp. 559-577, 2012.

[10] J. Wang, Q. Zong, R. Su, and B. L. Tian, "Continuous high order sliding mode controller design for a flexible airbreathing hypersonic vehicle," ISA Transactions, vol. 53, no. 3, pp. 690-698, 2014.

[11] Q. K. Shen, B. Jiang, and V. Cocquempot, "Fuzzy logic system-based adaptive fault-tolerant control for near-space vehicle attitude dynamics with actuator faults," IEEE Transactions on Fuzzy Systems, vol. 21, no. 2, pp. 289300, 2013.

[12] Y. Q. Huang, C. Y. Sun, C. S. Qian, J. M. Zhang, and L. Wang, "Polytopic LPV modeling and gain-scheduled switching control for a flexible air-breathing hypersonic vehicle," Journal of Systems Engineering and Electronics, vol. 24, no. 1, pp. 118127, 2003.

[13] Y. Q. Huang, C. Y. Sun, C. S. Qian, and L. Wang, "Non-fragile switching tracking control for a flexible air-breathing hypersonic vehicle based on polytopic LPV model," Chinese Journal of Aeronautics, vol. 26, no. 4, pp. 948-959, 2013.

[14] X. L. Shao and H. L. Wang, "Active disturbance rejection based trajectory linearization control for hypersonic reentry vehicle with bounded uncertainties," ISA Transactions, vol. 54, pp. 27-38, 2015.

[15] H. F. Sun, Z. L. Yang, and B. Meng, "Tracking control of a class of non-linear systems with applications to cruise control of air-breathing hypersonic vehicles," International Journal of Control, vol. 88, no. 5, pp. 885-896, 2015.

[16] J. Yang, S. H. Li, C. Y. Sun, and L. Guo, "Nonlineardisturbance-observer-based robust flight control for airbreathing hypersonic vehicles," IEEE Transactions on Aerospace and Electronic Systems, vol. 49, no. 2, pp. 1263-1275, 2013. 
[17] X. W. Bu, X. Y. Wu, Z. Ma, R. Zhang, and J. Q. Zhang, "Novel auxiliary error compensation design for the adaptive neural control of a constrained flexible air-breathing hypersonic vehicle," Neurocomputing, vol. 171, pp. 313324, 2016.

[18] D. X. Gao, S. X. Wang, and H. J. Zhang, "A singularly perturbed system approach to adaptive neural back-stepping control design of hypersonic vehicles," Journal of Intelligent \& Robotic Systems, vol. 73, no. 1-4, pp. 249-259, 2014.

[19] X. W. Bu, X. Y. Wu, R. Zhang, Z. Ma, and J. Q. Huang, "Tracking differentiator design for the robust backstepping control of a flexible air-breathing hypersonic vehicle," Journal of the Franklin Institute, vol. 352, no. 4, pp. 1739-1765, 2015.

[20] Q. Zong, F. Wang, R. Su, and S. K. Shao, "Robust adaptive backstepping tracking control for a flexible air-breathing hypersonic vehicle subject to input constraint," Proceedings of the Institution of Mechanical Engineers Part G: Journal of Aerospace Engineering, vol. 229, no. 1, pp. 10-25, 2014.

[21] X. W. Bu, X. Y. Wu, Y. X. Chen, and R. Y. Bai, "Design of a class of new nonlinear disturbance observers based on tracking differentiators for uncertain dynamic systems," International Journal of Control, Automation, and Systems, vol. 13, no. 3, pp. 595-602, 2015.

[22] D. Wang and C. Mu, "A novel neural optimal control framework with nonlinear dynamics: closed-loop stability and simulation verification," Neurocomputing, vol. 266, pp. 353-360, 2017.

[23] X. W. Bu, X. Y. Wu, M. Y. Tian, J. Q. Huang, R. Zhang, and Z. Ma, "High-order tracking differentiator based adaptive neural control of a flexible air-breathing hypersonic vehicle subject to actuators constraints," ISA Transactions, vol. 58, pp. 237-247, 2015.

[24] D. Wang, C. Mu, and D. Liu, "Data-driven nonlinear near-optimal regulation based on iterative neural dynamic programming," Acta Automatica Sinica, vol. 43, no. 3, pp. 366-375, 2017.

[25] X. W. Bu, X. Y. Wu, D. Z. Wei, and J. Q. Huang, "Neuralapproximation-based robust adaptive control of flexible air-breathing hypersonic vehicles with parametric uncertainties and control input constraints," Information Sciences, vol. 346-347, pp. 29-43, 2016.

[26] Q. Zong, F. Wang, B. L. Tian, and R. Su, "Robust adaptive dynamic surface control design for a flexible air-breathing hypersonic vehicle with input constraints and uncertainty," Nonlinear Dynamics, vol. 78, no. 1, pp. 289-315, 2014.

[27] W. A. Butt, L. Yan, and A. S. Kendrick, "Adaptive integral dynamic surface control of a hypersonic flight vehicle," International Journal of Systems Science, vol. 46, no. 10, pp. 17171728, 2015.

[28] B. Xu, "Robust adaptive neural control of flexible hypersonic flight vehicle with dead-zone input nonlinearity," Nonlinear Dynamics, vol. 80, no. 3, pp. 1509-1520, 2015.

[29] B. Xu and Y. Zhang, "Neural discrete back-stepping control of hypersonic flight vehicle with equivalent prediction model," Neurocomputing, vol. 154, pp. 337-346, 2015.

[30] X. W. Bu, X. Y. Wu, Z. Ma, and R. Zhang, "Nonsingular direct neural control of air-breathing hypersonic vehicle via backstepping," Neurocomputing, vol. 153, pp. 164-173, 2015.

[31] B. Xu, Y. H. Fan, and S. M. Zhang, "Minimal-learningparameter technique based adaptive neural control of hypersonic flight dynamics without back-stepping," Neurocomputing, vol. 164, pp. 201-209, 2015.

[32] M. A. Bolender and D. B. Doman, "Nonlinear longitudinal dynamical model of an air-breathing hypersonic vehicle," Journal of Spacecraft and Rockets, vol. 44, no. 2, pp. 374-387, 2007.

[33] J. T. Parker, A. Serrani, S. Yurkovich, M. A. Bolender, and D. B. Doman, "Control-oriented modeling of an air-breathing hypersonic vehicle," Journal of Guidance, Control, and Dynamics, vol. 30, no. 3, pp. 856-869, 2007.

[34] R. M. Sanner and J. E. Slotine, "Gaussian networks for direct adaptive control," IEEE Transactions on Neural Networks, vol. 3, no. 6, pp. 837-863, 1992.

[35] X. Wang, Z. Chen, and Z. Yuan, "Design and analysis for new discrete tracking-differentiators," Applied MathematicsA Journal of Chinese Universities Series B, vol. 18, no. 2, pp. 214-222, 2003.

[36] S. S. Ge, C. C. Hang, T. Lee, and T. Zhang, Stable Adaptive Neural Network Control, Kluwer Academic, USA, 2002.

[37] J. H. Park, S. H. Huh, S. H. Kim, S. J. Seo, and G. T. Park, "Direct adaptive controller for nonaffine nonlinear systems using self-structuring neural networks," IEEE Transactions on Neural Networks, vol. 16, no. 2, pp. 414-422, 2005.

[38] A. J. Calise, N. Hovakimyan, and M. Idan, "Adaptive output feedback control of nonlinear systems using neural networks," Automatica, vol. 37, no. 1, pp. 1201-1211, 2001. 


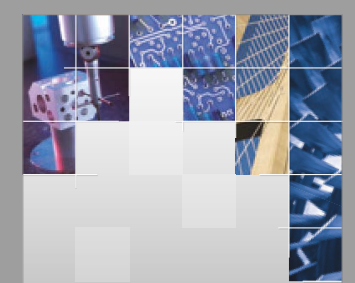

\section{Enfincering}
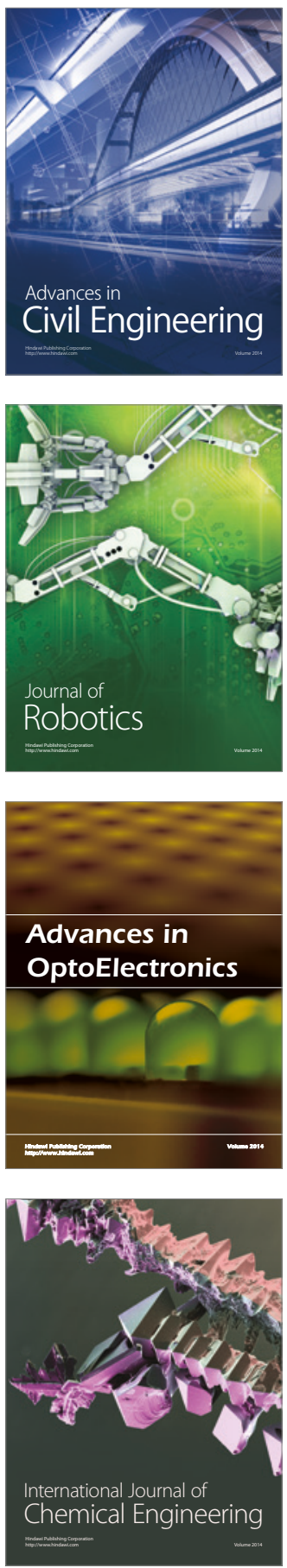

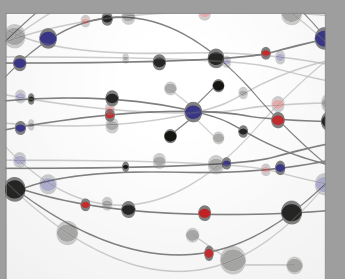

The Scientific World Journal

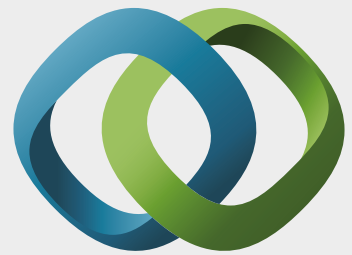

\section{Hindawi}

Submit your manuscripts at

https://www.hindawi.com
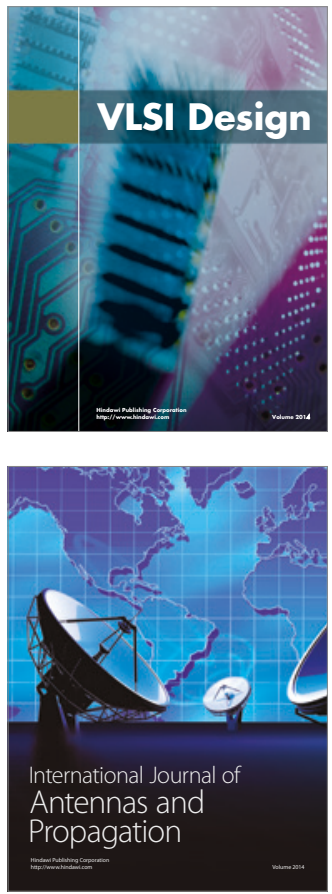

\section{Rotating}

Machinery
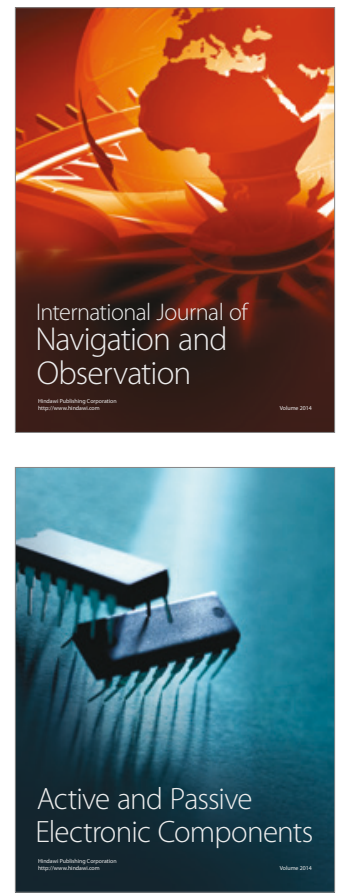
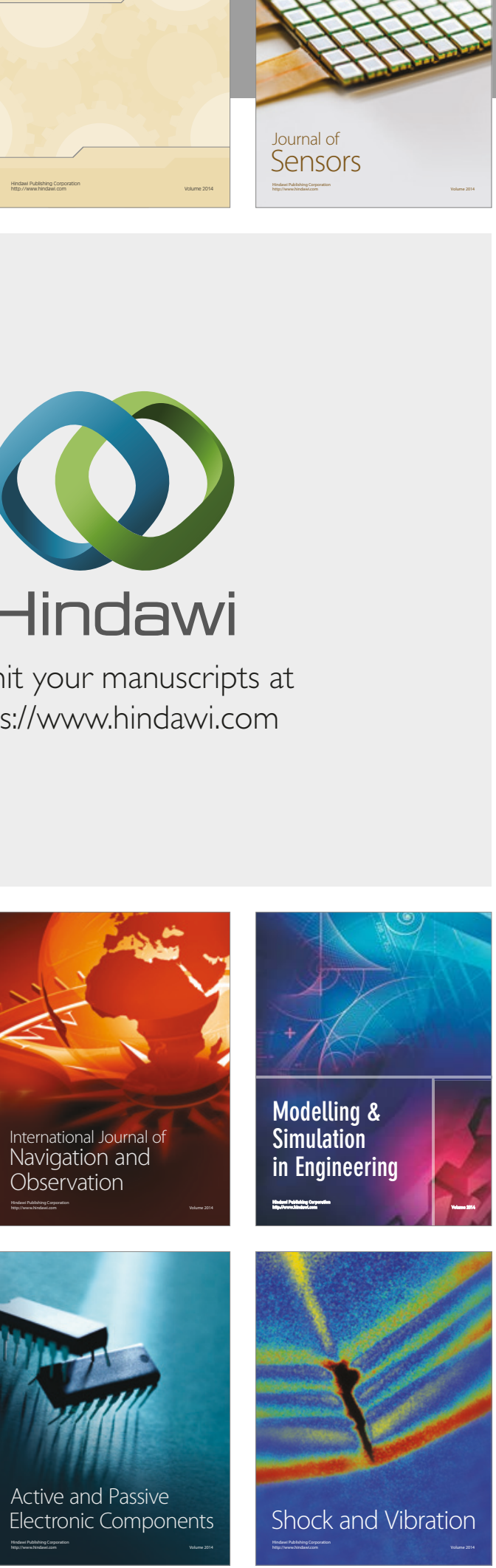
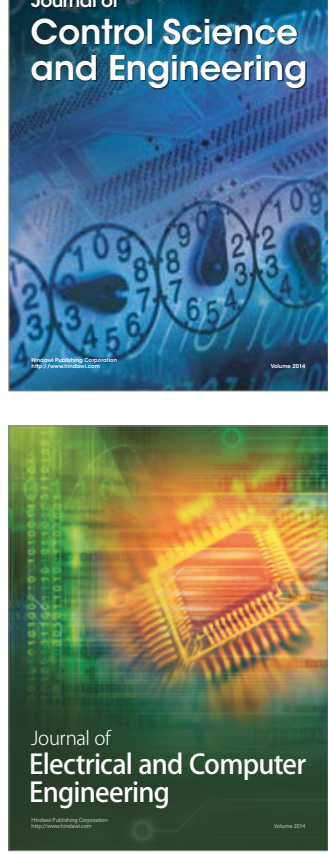

Distributed

Journal of

Control Science

and Engineering
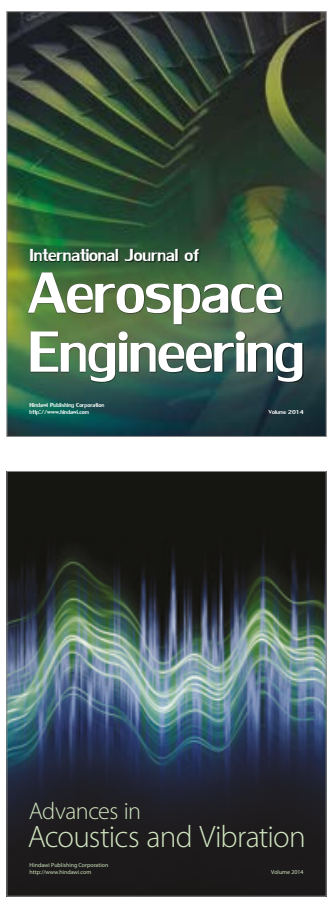

Sensor Networks 